\title{
HOMOGENEOUS KÄHLER AND SASAKIAN STRUCTURES RELATED TO COMPLEX HYPERBOLIC SPACES
}

\author{
P. M. GADEA ${ }^{1}$ AND J. A. OUBIÑA ${ }^{2}$ \\ ${ }^{1}$ Institute of Fundamental Physics, CSIC, \\ Serrano 113-bis, 28006 Madrid, Spain (pmgadea@iec.csic.es) \\ ${ }^{2}$ Departamento de Xeometría e Topoloxía, Universidade de Santiago de Compostela, \\ 15782 Santiago de Compostela, Spain (ja.oubina@usc.es)
}

\begin{abstract}
We study homogeneous Kähler structures on a non-compact Hermitian symmetric space and their lifts to homogeneous Sasakian structures on the total space of a principal line bundle over it, and we analyze the case of the complex hyperbolic space.

Keywords: Homogeneous Riemannian structures; homogeneous Kähler structures; homogeneous almost contact Riemannian manifolds; non-compact Hermitian symmetric spaces; Sasakian spaces
\end{abstract}

2000 Mathematics subject classification: Primary 53C30; Secondary 53C25, 53C35, 53C55

\section{Introduction}

The general theory of homogeneous Kähler manifolds is well-known, as well as the relation between homogeneous symplectic and homogeneous contact manifolds (see e.g. Boothby and Wang [6], Díaz Miranda and Reventós [9], and Dorfmeister and Nakajima [10]).

As is also widely known, a connected, simply connected and complete Riemannian manifold is a symmetric space if and only if its curvature tensor field is parallel. Ambrose and Singer [2] extended this result to obtain a characterization of homogeneous Riemannian manifolds in terms of the existence of a tensor field $S$ of type $(1,2)$ on the manifold, called a homogeneous Riemannian structure (see Tricerri and Vanhecke [26], where a classification of such structures is also given), satisfying certain properties (see (2.1); if $S=0$ one has the symmetric case). Moreover, Sekigawa [24] obtained the corresponding result for almost Hermitian manifolds, defining homogeneous almost Hermitian structures, which were classified by Abbena and Garbiero in [1] (among them the homogeneous Kähler structures). Its odd-dimensional version, the almost contact metric case, has been also studied (see, for instance, $[8,11,14,19]$ ).

In Section 2, we give the basic results about homogeneous Riemannian and homogeneous Kähler structures. In particular we consider these structures on Hermitian symmetric spaces of non-compact type. Besides the trivial homogeneous structure $S=0$ associated to the description of one such space as a symmetric space, other structures can be obtained associated to other descriptions as a homogeneous space and, in particular, to its description as a solvable Lie group given by an Iwasawa decomposition (§ 2.2). 
We also give a construction of homogeneous Sasakian structures on the bundle space of a principal line bundle over a Hermitian symmetric space of non-compact type, endowed with a connection 1-form that is the contact form of a Sasakian structure on the total space (Proposition 2.5).

The complex hyperbolic space $\mathbb{C H}(n)=S U(n, 1) / S(U(n) \times U(1))$ with the Bergman metric is an irreducible Hermitian symmetric space of non-compact type, and, up to homotheties, is the simply-connected complete complex space form of negative curvature. It has been characterized in [12] in terms of the existence of certain type of homogeneous Kähler structure on it, and in [7] a Lie-theoretical description of its homogeneous structure of linear type is found. In Section 3 we study the homogeneous Kähler structures on $\mathbb{C H}(n)$ from other point of view, which in particular provide an infinite number of descriptions of $\mathbb{C H}(n)$ as non-isomorphic solvable Lie groups. Moreover, we consider the principal line bundle over $\mathbb{C H}(n)$ with its Sasakian structure given in a natural way from a connection form on the bundle, and we obtain the families of homogeneous Sasakian structures on its bundle space following our previous general construction. Summarizing, we get:

(a) All the homogeneous Kähler structures on $\mathbb{C H}(n) \equiv A N$. They are given in terms of some 1-forms related by a system of differential equations on the solvable Lie group $A N$ (Theorem 3.1).

(b) The explicit description of a multi-parametric family of homogeneous Kähler structures on $\mathbb{C H}(n)$, given by using the generators of $\mathfrak{a}+\mathfrak{n}$ (Proposition 3.6), and the corresponding subgroups of the full isometry group $S U(n, 1)$ of $A N$ (Theorem 3.7).

(c) The explicit description of a one-parametric family of homogeneous Sasakian structures on the bundle space of the line bundle $\bar{M} \rightarrow \mathbb{C H}(n)$, given in terms of the horizontal lifts of the generators of $\mathfrak{a}+\mathfrak{n}$ and the fundamental vector field $\xi$ on $\bar{M}$ (Proposition 3.9 ), and their associated reductive decompositions (Propositions 3.11 and 3.12). One of them describes $\bar{M}$ as the complete simply connected $\varphi$-symmetric Sasakian space $\widehat{S U}(n, 1) / S U(n)$, which is also a Sasakian space form.

On the other hand, complex hyperbolic space was the first target spacetime where Nishino's [21] alternative (i.e., neither necessarily hyper-Kähler nor quaternion-Kähler) $N=(4,0)$ superstring theory proved to work. This model has some interesting features, among them, not to have (which is a trait common to heterotic $\sigma$-models) the incompatibility between the torsion tensor and quaternion-Kähler manifolds found by de Wit and van Nieuwenhuizen [27]. Another peculiarity is that in this case, one of the two scalars of the relevant global multiplet is promoted to coordinates on $\mathbb{C H}(n)$, while the other plays the role of a tangent vector under the holonomy group $S(U(n) \times U(1))$.

\section{Homogeneous Riemannian Structures}

Ambrose and Singer [2] proved that a connected, simply connected and complete Riemannian manifold is homogeneous if and only there exists a tensor field $S$ of type $(1,2)$ on $M$ such that the connection $\widetilde{\nabla}=\nabla-S$ satisfies the Eqs.

$$
\widetilde{\nabla} g=0, \quad \widetilde{\nabla} R=0, \quad \widetilde{\nabla} S=0,
$$


where $\nabla$ is the Levi-Civita connection of $g$ and $R$ its curvature tensor field, for which we adopt the conventions $R_{X Y} Z=\nabla_{[X, Y]} Z-\nabla_{X} \nabla_{Y} Z+\nabla_{Y} \nabla_{X} Z, R_{X Y Z W}=g\left(R_{X Y} Z, W\right)$. Such a tensor field $S$ is called a homogeneous Riemannian structure ([26]). We also denote by $S$ the associated tensor field of type $(0,3)$ on $M$ defined by $S_{X Y Z}=g\left(S_{X} Y, Z\right)$.

\subsection{Homogeneous Kähler structures}

An almost Hermitian manifold $(M, g, J)$ is said to be a homogeneous almost Hermitian manifold if there exists a Lie group of holomorphic isometries which acts transitively and effectively on $M$. Sekigawa proved the following

Theorem 2.1. ([24]) A connected, simply connected and complete almost Hermitian manifold $(M, g, J)$ is homogeneous if and only if there is a tensor field $S$ of type $(1,2)$ on $M$ which satisfies Eqs. (2.1) and $\widetilde{\nabla} J=0$.

A tensor $S$ satisfying the Eqs. (2.1) and $\widetilde{\nabla} J=0$ is called a homogeneous almost Hermitian structure. The almost Hermitian manifold $(M, g, J)$ is Kähler if and only if $J$ is integrable and the fundamental 2-form $\Omega$ on $M$, given by $\Omega(X, Y)=g(X, J Y)$, is closed, or equivalently $\nabla J=0$. In this case, a homogeneous almost Hermitian structure is also called a homogeneous Kähler structure, and we have

Proposition 2.2. A homogeneous Riemannian structure $S$ on a Kähler manifold $(M, g, J)$ is a homogeneous Kähler structure if and only if $S \cdot J=0$, or equivalently $S_{X Y Z}=$ $S_{X J Y J Z}$ for all the vector fields $X, Y, Z$ on $M$.

Corollary 2.3. A connected, simply connected and complete Kähler manifold $(M, g, J)$ is a homogeneous Kähler manifold if and only if there exists a homogeneous Kähler structure on $M$.

If $(M=G / H, g)$ is a homogeneous Riemannian manifold, where $G$ is a connected Lie group acting transitively and effectively on $M$ as a group of isometries and $H$ is the isotropy group at a point $o \in M$, then the Lie algebra $\mathfrak{g}$ of $G$ may be decomposed into a vector space direct sum $\mathfrak{g}=\mathfrak{h}+\mathfrak{m}$, where $\mathfrak{h}$ is the Lie algebra of $H$ and $\mathfrak{m}$ is an $\operatorname{Ad}(H)$-invariant subspace of $\mathfrak{g}$. If $G$ is connected and $M$ is simply connected then $H$ is connected, and the condition $\operatorname{Ad}(H) \mathfrak{m} \subset \mathfrak{m}$ is equivalent to $[\mathfrak{h}, \mathfrak{m}] \subset \mathfrak{m}$. The vector space $\mathfrak{m}$ is identified with $T_{o}(M)$ by the isomorphism $X \in \mathfrak{m} \rightarrow X_{o}^{*} \in T_{o}(M)$, where $X^{*}$ is the Killing vector field on $M$ generated by the one-parameter subgroup $\{\exp t X\}$ of $G$ acting on $M$. If $X \in \mathfrak{g}=\mathfrak{h}+\mathfrak{m}$, we write $X=X_{\mathfrak{h}}+X_{\mathfrak{m}},\left(X_{\mathfrak{h}} \in \mathfrak{h}, X_{\mathfrak{m}} \in \mathfrak{m}\right)$. The canonical connection $\widetilde{\nabla}$ of $M=G / H$ (with regard to the reductive decomposition $\mathfrak{g}=\mathfrak{h}+\mathfrak{m}$ ) is determined by

$$
\left(\widetilde{\nabla}_{X^{*}} Y^{*}\right)_{o}=\left[X^{*}, Y^{*}\right]_{o}=-[X, Y]_{o}^{*}=-\left([X, Y]_{\mathfrak{m}}\right)_{o}^{*}, \quad X, Y \in \mathfrak{m},
$$

and $S=\nabla-\widetilde{\nabla}$ satisfies the Ambrose-Singer Eqs. (2.1), and it is the homogeneous Riemannian structure associated to the reductive decomposition $\mathfrak{g}=\mathfrak{h}+\mathfrak{m}$. If $(M, g)$ is endowed with a compatible almost complex structure $J$ invariant by $G$ (so that $(M=G / H, g, J)$ is a homogeneous almost Hermitian manifold), restricting $J$ to $T_{o}(M) \equiv \mathfrak{m}$, we obtain a 
linear endomorphism $J_{o}$ of $\mathfrak{m}$ such that $J_{o}^{2}=-1$, and $J_{o} \operatorname{ad}_{\mathfrak{h}}=\operatorname{ad}_{\mathfrak{h}} J_{o}$. Moreover, $J$ is integrable if and only if

$$
\left[J_{o} X, J_{o} Y\right]_{\mathfrak{m}}-[X, Y]_{\mathfrak{m}}-J_{o}\left[X, J_{o} Y\right]_{\mathfrak{m}}-J_{o}\left[J_{o} X, Y\right]_{\mathfrak{m}}=0
$$

for all $X, Y \in \mathfrak{m}$ ([18], Ch. 10, Prop. 6.5).

Conversely, suppose that $(M, g)$ is a connected, simply connected and complete Riemannian manifold, and let $S$ be a homogeneous Riemannian structure on $(M, g)$. We put $\mathfrak{m}=T_{o}(M)$, where $o \in M$. If $\widetilde{R}$ is the curvature tensor of the connection $\widetilde{\nabla}=\nabla-S$, the holonomy algebra $\tilde{\mathfrak{h}}$ of $\widetilde{\nabla}$ is the Lie subalgebra of the Lie algebra of antisymmetric endomorphisms $\mathfrak{s o}(\mathfrak{m})$ of $\left(\mathfrak{m}, g_{o}\right)$ generated by the operators $\widetilde{R}_{X Y}$, where $X, Y \in \mathfrak{m}$. A Lie bracket is defined (Nomizu [20]) in the vector space direct sum $\tilde{\mathfrak{g}}=\tilde{\mathfrak{h}}+\mathfrak{m}$ by

$$
\begin{array}{llrl}
{[U, V]} & =U V-V U, & & U, V \in \tilde{\mathfrak{h}}, \\
{[U, X]=U(X),} & & U \in \tilde{\mathfrak{h}}, X \in \mathfrak{m}, \\
{[X, Y]=\widetilde{R}_{X Y}+S_{X} Y-S_{Y} X,} & & X, Y \in \mathfrak{m},
\end{array}
$$

and $\tilde{\mathfrak{g}}=\tilde{\mathfrak{h}}+\mathfrak{m}$ is the reductive decomposition corresponding to the homogeneous Riemannian structure $S$. Let $\widetilde{G}$ be the connected simply connected Lie group whose Lie algebra is $\tilde{\mathfrak{g}}$ and $\widetilde{H}$ the connected Lie subgroup of $\widetilde{G}$ whose Lie algebra is $\tilde{\mathfrak{h}}$. Then $\widetilde{G}$ acts transitively on $M$ as a group of isometries and $M$ is diffeomorphic to $\widetilde{G} / \widetilde{H}$. If $\Gamma$ is the set of the elements of $\widetilde{G}$ which act trivially on $M$, then $\Gamma$ is a discrete normal subgroup of $\widetilde{G}$, and the Lie group $G=\widetilde{G} / \Gamma$ acts transitively and effectively on $M$ as a group of isometries, with isotropy group $H=\widetilde{H} / \Gamma$. Then $M$ is diffeomorphic to $G / H$. Now, if $J$ is a compatible almost complex structure on $(M, g)$ and $S$ is a homogeneous almost Hermitian structure, then the holonomy algebra $\tilde{\mathfrak{h}}$ is a subalgebra of the Lie algebra $\mathfrak{u}(\mathfrak{m})=\{A \in \mathfrak{s o}(\mathfrak{m}): A \cdot J=0\}$ of the unitary group, and $M \approx \widetilde{G} / \widetilde{H} \approx G / H$ is a homogeneous almost Hermitian manifold.

\subsection{Hermitian symmetric spaces of non-compact type}

Suppose that $(M=G / K, g, J)$ is a connected Hermitian symmetric space of non-compact type, where $G=I_{0}(M)$ is the identity component of the group of (holomorphic) isometries and $K$ is a maximal compact subgroup of $G$. Then $M$ is simply connected and the Hermitian structure is Kähler. We consider a Cartan decomposition $\mathfrak{g}=\mathfrak{k}+\mathfrak{p}$ of the Lie algebra $\mathfrak{g}$ of $G$, and the Iwasawa decomposition $\mathfrak{g}=\mathfrak{k}+\mathfrak{a}+\mathfrak{n}$, where $\mathfrak{k}$ is the Lie algebra of $K, \mathfrak{a} \subset \mathfrak{p}$ is a maximal $\mathbb{R}$-diagonalizable subalgebra of $\mathfrak{g}$, and $\mathfrak{n}$ is a nilpotent subalgebra. Let $A$ and $N$ be the connected abelian and nilpotent Lie subgroups of $G$ whose Lie algebras are $\mathfrak{a}$ and $\mathfrak{n}$, respectively. The solvable Lie group $A N$ acts simply transitively on $M$, so $M$ is isometric to $A N$ equipped with the left-invariant Riemannian metric defined by the scalar product $\langle$, $\rangle$, induced on $\mathfrak{a}+\mathfrak{n} \cong \mathfrak{g} / \mathfrak{k} \cong \mathfrak{p}$ by a positive multiple of $B_{\mid \mathfrak{p} \times \mathfrak{p}}$, where $B$ is the Killing form of $\mathfrak{g}$.

Now, let $\hat{G}$ be a connected closed Lie subgroup of $G$ which acts transitively on $M$. The isotropy group of this action at $o=K \in M$ is $H=\hat{G} \cap K$. Then $M=G / K$ has also the description $M \equiv \hat{G} / H$, and $o \equiv H \in \hat{G} / H$. Let $\hat{\mathfrak{g}}=\mathfrak{h}+\mathfrak{m}$ be a reductive decomposition of the Lie algebra $\hat{\mathfrak{g}}$ of $\hat{G}$ corresponding to $M \equiv \hat{G} / H$. 
We have the isomorphisms of vector spaces

$$
\mathfrak{p} \cong \mathfrak{g} / \mathfrak{k} \cong \hat{\mathfrak{g}} / \mathfrak{h} \cong \mathfrak{m} \cong T_{o}(M) \cong \mathfrak{a}+\mathfrak{n},
$$

with

$$
\xi: \mathfrak{p} \stackrel{\cong}{\rightrightarrows} \mathfrak{m}, \quad \mu: \mathfrak{m} \cong T_{o}(M), \quad \zeta: T_{o}(M) \stackrel{\cong}{\rightrightarrows} \mathfrak{a}+\mathfrak{n},
$$

given by

$$
\xi^{-1}(Z)=Z_{\mathfrak{p}}, \quad \mu(Z)=Z_{o}^{*}, \quad \zeta^{-1}(X)=X_{o}^{*}, \quad Z \in \mathfrak{m}, X \in \mathfrak{a}+\mathfrak{n} .
$$

For each $X \in \mathfrak{g}$, we have $\left(X_{\mathfrak{k}}\right)_{o}^{*}=0$ and $\left(\nabla\left(X_{\mathfrak{p}}\right)^{*}\right)_{o}=0$, and since the Levi-Civita connection $\nabla$ has no torsion, for each $X, Y \in \mathfrak{g}$, we have

$$
\left(\nabla_{X^{*}} Y^{*}\right)_{o}=\left(\nabla_{\left(X_{\mathfrak{p}}\right)^{*}}\left(Y_{\mathfrak{k}}\right)^{*}\right)_{o}=\left[\left(X_{\mathfrak{p}}\right)^{*},\left(Y_{\mathfrak{k}}\right)^{*}\right]_{o}=-\left[X_{\mathfrak{p}}, Y_{\mathfrak{k}}\right]_{o}^{*} .
$$

The reductive decomposition $\hat{\mathfrak{g}}=\mathfrak{h}+\mathfrak{m}$ defines the homogeneous Riemannian structure $S=\nabla-\widetilde{\nabla}$, where $\widetilde{\nabla}$ is the canonical connection of $M \equiv \hat{G} / H$ with respect to $\hat{\mathfrak{g}}=\mathfrak{h}+\mathfrak{m}$, which is $\hat{G}$-invariant and uniquely determined by $\left(\widetilde{\nabla}_{X^{*}} Y^{*}\right)_{o}=-[X, Y]_{o}^{*}$, for $X, Y \in \mathfrak{m}(2.2)$. The tensor field $S$ is also uniquely determined by its value at $o$ because $M \equiv \hat{G} / H$ and $S$ is $\hat{G}$-invariant. Since $J$ is $\hat{G}$-invariant, from [18], Ch. 10, Prop. 2.7, it follows that $\widetilde{\nabla} J=0$, and by Theorem 2.1,S is a homogeneous Kähler structure.

We have

$$
\left(S_{X^{*}} Y^{*}\right)_{o}=\left(\nabla_{X^{*}} Y^{*}\right)_{o}+[X, Y]_{o}^{*}=\nabla_{Y_{o}^{*}} X^{*}, \quad X, Y \in \mathfrak{m} .
$$

By (2.4) and (2.5), $S$ is given by

$$
S_{X_{o}^{*}} Y_{o}^{*}=\left[X_{\mathfrak{k}}, Y_{\mathfrak{p}}\right]_{o}^{*}, \quad X, Y \in \mathfrak{m} .
$$

Then, for each $X, Y \in \mathfrak{a}+\mathfrak{n}$, we have

$$
S_{X_{o}^{*}} Y_{o}^{*}=S_{\xi\left(X_{\mathfrak{p}}\right)_{o}^{*}} \xi\left(Y_{\mathfrak{p}}\right)_{o}^{*}=\left[\left(\xi\left(X_{\mathfrak{p}}\right)\right)_{\mathfrak{e}}, Y_{\mathfrak{p}}\right]_{o}^{*} .
$$

The complex structure $J$ on $M=G / K$ is defined by an element $E_{J}$ in the center of $\mathfrak{k}$, and it defines the complex structure $J \in \operatorname{End}(\mathfrak{a}+\mathfrak{n})$ such that the following diagram is commutative, and $(\mathfrak{a}+\mathfrak{n},\langle\rangle, J$,$) becomes a Hermitian vector space isomorphic to$ $\left(T_{o}(M), g_{o}, J_{o}\right)$

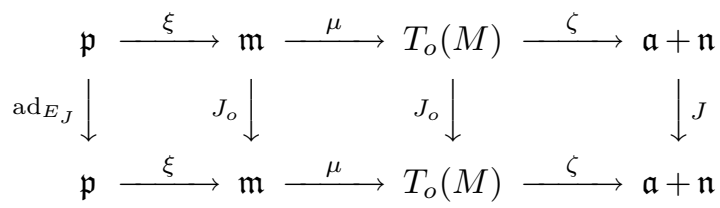

Let $A$ and $N$ be the connected abelian and nilpotent Lie subgroups of $G$ whose Lie algebras are $\mathfrak{a}$ and $\mathfrak{n}$, respectively. The solvable Lie group $A N$ acts simply transitively on $M$. Then $M$ is isometric to $A N$ equipped with the left-invariant Riemannian metric defined by the scalar product induced on $\mathfrak{a}+\mathfrak{n} \cong \mathfrak{g} / \mathfrak{k} \cong \mathfrak{p}$ by a positive multiple of $B_{\mid \mathfrak{p} \times \mathfrak{p}}$, where $B$ is the Killing form of $\mathfrak{g}$, so that $A N$ equipped with the left-invariant almost complex structure defined by $J$ is a Kähler manifold. 


\subsection{Homogeneous almost contact Riemannian manifolds}

An almost contact structure on a $(2 n+1)$-dimensional manifold $\bar{M}$ is a triple $(\varphi, \xi, \eta)$, where $\varphi$ is a tensor field of type $(1,1), \xi$ a vector field (called the characteristic vector field) and $\eta$ a differential 1 -form on $\bar{M}$ such that

$$
\varphi^{2}=-\mathrm{id}+\eta \otimes \xi, \quad \eta(\xi)=1 .
$$

Then $\varphi \xi=0, \eta \circ \varphi=0$, and $\varphi$ has rank $2 n$. If $\bar{g}$ is a Riemannian metric on $\bar{M}$ such that $\bar{g}(\varphi \tilde{X}, \varphi \tilde{Y})=\bar{g}(\tilde{X}, \tilde{Y})-\eta(\tilde{X}) \eta(\tilde{Y})$ for all vector fields $\tilde{X}$ and $\tilde{Y}$ on $\bar{M}$ then $(\varphi, \xi, \eta, \bar{g})$ is said to be an almost contact metric structure on $\bar{M}$. In this case, $\bar{g}(\tilde{X}, \xi)=\eta(\tilde{X})$. The 2 -form $\Phi$ on $M$ defined by $\Phi(\tilde{X}, \tilde{Y})=\bar{g}(\tilde{X}, \varphi \tilde{Y})$ is called the fundamental 2 -form of the almost contact metric structure $(\varphi, \xi, \eta, \bar{g})$. If $d \eta(\tilde{X}, \tilde{Y})=\tilde{X} \eta(\tilde{Y})-\tilde{Y} \eta(\tilde{X})-\eta([\tilde{X}, \tilde{Y}])=$ $2 \Phi(\tilde{X} \tilde{Y})$, then $(\phi, \xi, \eta, \bar{g})$ is called a contact metric (or contact Riemannian) structure; in particular, $\eta \wedge(d \eta)^{n} \neq 0$, that is, $\eta$ is a contact form on $\bar{M}$. If

$$
\left(D_{\tilde{X}} \varphi\right) \tilde{Y}=\bar{g}(\tilde{X}, \tilde{Y}) \xi-\eta(\tilde{Y}) \tilde{X}
$$

where $D$ is the Levi-Civita connection of $\bar{g}$, then $(\varphi, \xi, \eta, \bar{g})$ is called a Sasakian structure, and the manifold $\bar{M}$ with such a structure is a Sasakian manifold. Sasakian manifolds can also be characterized as normal contact metric manifolds and they are in some sense odd-dimensional analogues of Kähler manifolds (see Blair [3, 4]).

If $(\varphi, \xi, \eta, \bar{g})$ is an almost contact metric structure on $\bar{M}$ and $(\bar{M}=\bar{G} / H, \bar{g})$ is a homogeneous Riemannian manifold such that $\varphi$ is invariant under the action of the connected Lie group $\bar{G}$ (and hence so are $\xi$ and $\eta$ ) then $(\bar{M}, \varphi, \xi, \eta, \bar{g})$ is called a homogeneous almost contact Riemannian manifold $([8,14,19])$. Let $\bar{R}$ be the curvature tensor field of the Levi-Civita connection $D$ of $\bar{g}$. Let $S$ be a homogeneous Riemannian structure on $\bar{M}$, that is $\widetilde{D} \bar{g}=0, \widetilde{D} \bar{R}=0$ and $\widetilde{D} S=0$, where $\widetilde{D}=D-S$. If $S$ satisfies the additional condition $\widetilde{D} \varphi=0$ (and hence $\widetilde{D} \xi=0$ and $\widetilde{D} \eta=0$ ), then $S$ is called a homogeneous almost contact metric structure on $(\bar{M}, \varphi, \xi, \eta, \bar{g})$. From the results of Kiričenko in [17] on homogeneous Riemannian spaces with invariant tensor structure, it follows

Theorem 2.4. A connected, simply connected and complete almost contact metric manifold $(\bar{M}, \varphi, \xi, \eta, \bar{g})$ is a homogeneous almost contact Riemannian manifold if and only if there exists a homogeneous almost contact metric structure on $\bar{M}$.

A homogeneous almost contact metric structure on a Sasakian manifold will be also called a homogeneous Sasakian structure.

\subsection{Principal 1-bundles over almost Hermitian manifolds}

Let $(M, g, J)$ be an almost Hermitian manifold and let $\bar{M}$ be the bundle space of a principal 1-bundle over $M$. Let $\eta$ be a connection (form) on the principal bundle $\pi: \bar{M} \rightarrow M$, and let $\xi$ be the fundamental vector field on $\bar{M}$ defined by the element 1 of the Lie algebra $\mathbb{R}$ of the structure group of the bundle. Then $\eta(\xi)=1$. For each vector field $X$ on $M$, we denote by $X^{H}$ the horizontal lift of $X$ with respect to $\eta$. If $\bar{X}$ is a vector field on $\bar{M}$, its vertical part is $\eta(\bar{X}) \xi$. Then, for any vector fields $X$ and $Y$ on $M$, we have

$$
\left[X^{H}, Y^{H}\right]=[X, Y]^{H}+\eta\left(\left[X^{H}, Y^{H}\right]\right) \xi .
$$


Moreover, $\left[X^{H}, \xi\right]=0$, because $X^{H}$ is invariant under the action of the structural group. We define a tensor field $\varphi$ of type $(1,1)$ and a Riemannian metric $\bar{g}$ on $\bar{M}$ by

$$
\varphi X^{H}=(J X)^{H}, \varphi \xi=0, \quad \bar{g}=\pi^{*} g+\eta \otimes \eta,
$$

where $X$ and $Y$ are vector fields on $M$. Clearly, $(\varphi, \xi, \eta, \bar{g})$ is an almost contact metric structure on $\bar{M}$, and we have $\bar{g}\left(X^{H}, Y^{H}\right)=g(X, Y) \circ \pi$, and $\bar{g}\left(X^{H}, \xi\right)=0$. Let $\Phi$ be its 2 -fundamental form. If $\Omega$ is the fundamental 2-form of the almost Hermitian manifold $(M, g, J)$, then $\pi^{*} \Omega=\Phi$. $[22])$

If $\nabla$ and $D$ are the the Levi-Civita connections of $g$ and $\bar{g}$, respectively, then (Ogiue

$$
D_{X^{H}} Y^{H}=\left(\nabla_{X} Y\right)^{H}+\frac{1}{2} \eta\left(\left[X^{H}, Y^{H}\right]\right) \xi=\left(\nabla_{X} Y\right)^{H}-\frac{1}{2} d \eta\left(X^{H}, Y^{H}\right) \xi,
$$

and $D_{X^{H}} \xi=D_{\xi} X^{H}=-\varphi X^{H}$. Now, if $2 \Phi=d \eta$, Eq. (2.6) is satisfied as one can easily see by replacing $(\tilde{X}, \tilde{Y})$ by $\left(X^{H}, Y^{H}\right),\left(X^{H}, \xi\right)$, and $\left(\xi, Y^{H}\right)$, respectively. Then, if the almost contact metric structure $(\varphi, \xi, \eta, \bar{g})$ is a contact structure, it is also Sasakian.

Suppose now that the structural group of the principal 1-bundle $\pi: \bar{M} \rightarrow M$ is $\mathbb{R}$ and that the base manifold is a $2 n$-dimensional connected Hermitian symmetric space of noncompact type $(M=G / K, g, J)$, so that $M$ is isometric to the solvable Lie group $A N$ as in $\S 2.2$. Then $M$ is holomorphically diffeomorphic to a bounded symmetric domain, i.e., to a simply connected open subset of $\mathbb{C}^{n}$ such that each point is an isolated fixed point of an involutive holomorphic diffeomorphism of itself ([15], Ch. VIII, Th. 7.1). Since $\pi: \bar{M} \rightarrow M$ is a principal line bundle over the paracompact manifold $M$, then it admits a global section ([18], Ch. I, Th. 5.7), so there exists a diffeomorphism $\bar{M} \rightarrow M \times \mathbb{R}$, and the bundle space $\bar{M}$ may be identified with $A N \times \mathbb{R}$, with $\pi$ being the projection on $A N$. On the other hand, since the fundamental 2-form $\Omega$ associated to the Kähler structure $(g, J)$ is closed, $\Omega=d \zeta$ for some real analytic 1-form $\zeta$ on $A N$. We consider the connection form $\eta=2 \pi^{*} \zeta+d t$ on $\bar{M}$, where $t$ is the coordinate of $\mathbb{R}$. The vertical vector field $\xi$ with $\eta(\xi)=1$ can be identified with $\frac{d}{d t}$, and we consider $\varphi$ and $\bar{g}$ given by (2.7). Then $2 \Phi=2 \pi^{*} \Omega=2 \pi^{*} d \zeta=d \eta$, and hence $(\varphi, \xi, \eta, \bar{g})$ is a Sasakian structure on $\bar{M}$.

If $\bar{S}$ is a homogeneous almost contact metric structure on $\bar{M}$, and $\widetilde{D}=D-\bar{S}$, then $\widetilde{D} \xi=0$, and hence $\bar{S}_{X^{H}} \xi=D_{X^{H}} \xi=-\varphi X^{H}$. We have

Proposition 2.5. Let $(M=G / K, g, J)$ be a connected Hermitian symmetric space of non-compact type. Let $\pi: \bar{M} \rightarrow M$ be a principal line bundle with connection form $\eta$ such that the almost contact metric structure $(\varphi, \xi, \eta, \bar{g})$ on $\bar{M}$ defined by (2.7) is Sasakian.

(a) If $S$ is a homogeneous Kähler structure on $M$ then the tensor field $\bar{S}$ on $\bar{M}$ defined by

$$
\bar{S}_{X^{H}} Y^{H}=\left(S_{X} Y\right)^{H}-\bar{g}\left(X^{H}, \varphi Y^{H}\right) \xi, \quad \bar{S}_{X^{H}} \xi=-\varphi X^{H}=\bar{S}_{\xi} X^{H}, \quad \bar{S}_{\xi} \xi=0,
$$

for all vector fields $X$ and $Y$ on $M$, is a homogeneous Sasakian structure on $\bar{M}$.

(b) $\left\{S^{t}: t \in \mathbb{R}\right\}$ defined by

$$
S_{X^{H}}^{t} Y^{H}=-\bar{g}\left(X^{H}, \varphi Y^{H}\right) \xi, \quad S_{X^{H}}^{t} \xi=-\varphi X^{H}, \quad S_{\xi}^{t} X^{H}=-t \varphi X^{H}, \quad S_{\xi}^{t} \xi=0,
$$

is a family of homogeneous Sasakian structures on $\bar{M}$. 
Proof. (a) If $\widetilde{D}=D-\bar{S}$, then since $\bar{S}_{X^{H} Y^{H} Z^{H}}=\bar{g}\left(\left(S_{X} Y\right)^{H}, Z^{H}\right)=g\left(S_{X} Y, Z\right) \circ \pi=$ $-g\left(Y, S_{X} Z\right) \circ \pi=-\bar{g}\left(Y^{H},\left(S_{X} Z\right)^{H}\right)=-\bar{S}_{X^{H} Z^{H} Y^{H}}$, and $\bar{S}_{X^{H} Y^{H} \xi}=-\bar{S}_{X^{H} \xi Y^{H}}$, the condition $\widetilde{D} \bar{g}=0$ is satisfied. On the other hand, if $\widetilde{\nabla}=\nabla-S$ we have

$$
\widetilde{D}_{X^{H}} Y^{H}=\left(\widetilde{\nabla}_{X} Y\right)^{H}, \quad \widetilde{D}_{X^{H}} \xi=\widetilde{D}_{\xi} X^{H}=0 .
$$

We can identify $M=G / K$ with the solvable Lie group $A N$ in an Iwasawa decomposition $G=K A N$ and consider the Lie algebra $\mathfrak{a}+\mathfrak{n}$ of $A N$. If $\tilde{U}, \tilde{V}, \tilde{X}, \tilde{Y}, \tilde{Z}$ are horizontal lifts of elements of $\mathfrak{a}+\mathfrak{n}$ or some of them are the vertical vector field $\xi$, then

$$
\left(\widetilde{D}_{\tilde{U}} \bar{R}\right)_{\tilde{X} \tilde{Y} \tilde{Z} \tilde{V}}=-\bar{R}_{\tilde{X} \tilde{Y} \tilde{Z} \widetilde{D}_{\tilde{U}} \tilde{V}}+\bar{R}_{\tilde{X} \tilde{Y} \tilde{V} \widetilde{D}_{\tilde{U}} \tilde{Z}}-\bar{R}_{\tilde{Z} \tilde{V} \tilde{X} \widetilde{D}_{\tilde{U}} \tilde{Y}}+\bar{R}_{\tilde{Z} \tilde{V} \tilde{Y} \widetilde{D}_{\tilde{U}} \tilde{X}}
$$

since $\tilde{U}\left(\bar{R}_{\tilde{X} \tilde{Y} \tilde{Z} \tilde{V}}\right)=0$. Now, if $X, Y, Z, V \in \mathfrak{a}+\mathfrak{n}$, then

$$
\begin{aligned}
\bar{R}_{X^{H} Y^{H} Z^{H} V^{H}}= & \left(R_{X Y Z V}-2 g(X, J Y) g(Z, J V)\right. \\
& +g(X, J V) g(Y, J Z)-g(X, J Z) g(Y, J V)) \circ \pi, \\
\bar{R}_{X^{H} Y^{H} Z^{H} \xi}= & -\bar{g}\left([X, Y]^{H}, \varphi Z^{H}\right) \\
& +\bar{g}\left(\left(\nabla_{X} Z\right)^{H}, \varphi Y^{H}\right)-\bar{g}\left(\left(\nabla_{Y} Z\right)^{H}, \varphi X^{H}\right), \\
\bar{R}_{X^{H} \xi Z^{H} \xi}= & \bar{g}\left(D_{X^{H}} \xi, D_{Z^{H}} \xi\right) .
\end{aligned}
$$

By using (2.8) and (2.10), the conditions $\widetilde{\nabla} R=0$ and $\widetilde{\nabla} J=0$ for the homogeneous Kähler structure $S$ on $M$, and the formula $\bar{R}_{\tilde{X} \tilde{Y}} \xi=\eta(\tilde{X}) \tilde{Y}-\eta(\tilde{Y}) \tilde{X}$ for the Sasakian manifold $(\bar{M}, \varphi, \xi, \eta, \bar{g})$ ([4], Prop. 7.3), one obtains from (2.9) that $\widetilde{D} \bar{R}=0$. Now, $\left(\widetilde{D}_{U^{H}} \bar{S}\right)_{X^{H}} Y^{H}=\left(\left(\widetilde{\nabla}_{U} S\right)_{X} Y\right)^{H},\left(\widetilde{D}_{U^{H}} \bar{S}\right)_{X^{H}} \xi=-\left(\left(\widetilde{\nabla}_{U} J\right) X\right)^{H}$, and $\widetilde{D}_{\xi} S=0$, then $\widetilde{D} S=0$. Moreover, $\left(\widetilde{D}_{X^{H}} \varphi\right) Y^{H}=\left(\left(\widetilde{\nabla}_{X} J\right) Y\right)^{H}$ and $\left(\widetilde{D}_{X^{H}} \varphi\right) \xi=0$, then $\widetilde{D} \varphi=0$, and $\bar{S}$ is a homogeneous Sasakian structure on $\bar{M}$.

(b) If $t=1$ the corresponding tensor $S^{1}$ coincides with $\bar{S}$ in (a) for $S=0$. For arbitrary $t$, if $\widetilde{D}^{t}=D-S^{t}$ we have $\widetilde{D}_{\xi}^{t} X^{H}=(t-1)(J X)^{H}$, and we get $\widetilde{D}^{t} \bar{g}=0$, $\widetilde{D}^{t} \bar{R}=0, \widetilde{D}^{t} \bar{S}^{t}=0, \widetilde{D}^{t} \varphi=0$.

\section{The Complex Hyperbolic Space $\mathbb{C H}(n)$}

\section{1 $\mathbb{C H}(n)$ as a solvable Lie group}

The complex hyperbolic space $\mathbb{C H}(n)$, which may be identified with the unit ball in $\mathbb{C}^{n}$ endowed with the hyperbolic metric of constant holomorphic sectional curvature -4 , may also be viewed as the irreducible Hermitian symmetric space of non-compact type $S U(n, 1) / S(U(n) \times U(1))$.

The Lie algebra $\mathfrak{s u}(n, 1)$ of $S U(n, 1)$ can be described as the subalgebra of $\mathfrak{s l}(n+1, \mathbb{C})$ of all matrices of the form

$$
X=\left(\begin{array}{cc}
Z & P^{T} \\
\bar{P} & i c
\end{array}\right)
$$


where $Z \in \mathfrak{u}(n), c \in \mathbb{R}$, and $P=\left(p_{1}, \ldots, p_{n}\right) \in \mathbb{C}^{n}$. The involution $\tau$ of $\mathfrak{s u}(n, 1)$ given by $\tau(X)=-\bar{X}^{T}$ defines the Cartan decomposition $\mathfrak{s u}(n, 1)=\mathfrak{k}+\mathfrak{p}$, where

$$
\mathfrak{k}=\left\{\left(\begin{array}{cc}
Z & 0 \\
0 & i c
\end{array}\right): \operatorname{tr} Z+i c=0\right\} \cong \mathfrak{s}(\mathfrak{u}(n) \oplus \mathfrak{u}(1)), \quad \mathfrak{p}=\left\{\left(\begin{array}{cc}
0 & P^{T} \\
\bar{P} & 0
\end{array}\right)\right\}
$$

The element $A_{0}$ of $\mathfrak{p}$ defined by $P=(0, \ldots, 0,1)$ generates a maximal $\mathbb{R}$-diagonalizable subalgebra $\mathfrak{a}$ of $\mathfrak{s u}(n, 1)$. Let $f_{0}$ be the linear functional on $\mathfrak{a}$ given by $f_{0}\left(A_{0}\right)=1$. If $n>1$, the set of roots of $(\mathfrak{s u}(n, 1), \mathfrak{a})$ is $\Sigma=\left\{ \pm f_{0}, \pm 2 f_{0}\right\}$, the set $\Pi=\left\{f_{0}\right\}$ is a system of simple roots, and the corresponding positive root system is $\Sigma^{+}=\left\{f_{0}, 2 f_{0}\right\}$. If $n=1$, $\Sigma=\left\{ \pm 2 f_{0}\right\}$, and $\Pi=\Sigma^{+}=\left\{2 f_{0}\right\}$.

Let $E_{i j}$ be the matrix in $\mathfrak{g l}(n, \mathbb{C})$ such that the entry at the $i$-th row and the $j$-th column is 1 and the other entries are all zero. The root vector spaces are

$$
\begin{array}{rll}
\mathfrak{g}_{f_{0}}=\left\langle Z_{j}, Z_{j}^{\prime}: 1 \leqslant j \leqslant n-1\right\rangle & \text { (if } n>1), & \mathfrak{g}_{2 f_{0}}=\langle U\rangle, \\
\mathfrak{g}_{-f_{0}}=\left\langle W_{j}, W_{j}^{\prime}: 1 \leqslant j \leqslant n-1\right\rangle & \text { (if } n>1), & \mathfrak{g}_{-2 f_{0}}=\langle V\rangle,
\end{array}
$$

where

$$
\begin{aligned}
Z_{j} & =E_{j n}-E_{j, n+1}-E_{n j}-E_{n+1, j}, \quad Z_{j}^{\prime}=i\left(E_{j n}-E_{j, n+1}+E_{n j}+E_{n+1, j}\right), \\
W_{j} & =E_{j n}+E_{j, n+1}-E_{n j}+E_{n+1, j}, \quad W_{j}^{\prime}=i\left(E_{j n}+E_{j, n+1}+E_{n j}-E_{n+1, j}\right), \\
U & =i\left(E_{n n}-E_{n, n+1}+E_{n+1, n}-E_{n+1, n+1}\right), \\
V & =i\left(E_{n n}+E_{n, n+1}-E_{n+1, n}-E_{n+1, n+1}\right) .
\end{aligned}
$$

If $n>2$, the centralizer of $\mathfrak{a}$ in $\mathfrak{k}$ is $Z_{\mathfrak{k}}(\mathfrak{a})=\left\langle C_{r}, F_{j k}, H_{j k}: r, j, k=1, \ldots, n-1\right.$, $j<k\rangle \cong \mathfrak{u}(n-1)$, where

$$
C_{r}=2 i E_{r r}-i E_{n n}-i E_{n+1, n+1}, \quad F_{j k}=E_{j k}-E_{k j}, \quad H_{j k}=i\left(E_{j k}+E_{k j}\right)
$$

and $\mathfrak{s u}(n, 1)=\left(Z_{\mathfrak{k}}(\mathfrak{a})+\mathfrak{a}\right)+\sum_{f \in \Sigma} \mathfrak{g}_{f}$ is the restricted-root space decomposition. We also have the Iwasawa decomposition $\mathfrak{s u}(n, 1)=\mathfrak{k}+\mathfrak{a}+\mathfrak{n}$, where $\mathfrak{n}=\mathfrak{g}_{f_{0}}+\mathfrak{g}_{2 f_{0}}=\left\langle U, Z_{j}, Z_{j}^{\prime}\right.$ : $1 \leqslant j \leqslant n-1\rangle$.

If $n=2$, we put $C=C_{1}=\operatorname{diag}(2 i,-i,-i), Z=Z_{1}, Z^{\prime}=Z_{1}^{\prime}$, and in this case $C$ generates $Z_{\mathfrak{k}}(\mathfrak{a})$, and $\mathfrak{a}+\mathfrak{n}=\left\langle A_{0}, U, Z, Z^{\prime}\right\rangle$. If $n=1, Z_{\mathfrak{k}}(\mathfrak{a})=0$, we have the restrictedroot space decomposition $\mathfrak{s u}(1,1)=\mathfrak{a}+\left(\mathfrak{g}_{2 f_{0}}+\mathfrak{g}_{-2 f_{0}}\right)=\left\langle A_{0}\right\rangle+\langle U, V\rangle$, and the solvable part in the Iwasawa decomposition is $\mathfrak{a}+\mathfrak{n}=\left\langle A_{0}, U\right\rangle$.

By using the Cartan decomposition $\mathfrak{s u}(n, 1)=\mathfrak{k}+\mathfrak{p}$, we express each element $X \in$ $\mathfrak{s u}(n, 1)$ as the sum $X=X_{\mathfrak{k}}+X_{\mathfrak{p}}\left(X_{\mathfrak{k}} \in \mathfrak{k}, X_{\mathfrak{p}} \in \mathfrak{p}\right)$. In particular, we have

$$
\begin{aligned}
U_{\mathfrak{k}} & =i\left(E_{n n}-E_{n+1, n+1}\right), & U_{\mathfrak{p}} & =i\left(E_{n+1, n}-E_{n, n+1}\right), \\
\left(Z_{j}\right)_{\mathfrak{k}} & =E_{j n}-E_{n j}, & \left(Z_{j}\right)_{\mathfrak{p}} & =-\left(E_{n+1, j}+E_{j, n+1}\right), \\
\left(Z_{j}^{\prime}\right)_{\mathfrak{k}} & =i\left(E_{j n}+E_{n j}\right), & \left(Z_{j}^{\prime}\right)_{\mathfrak{p}} & =i\left(E_{n+1, j}-E_{j, n+1}\right) .
\end{aligned}
$$

From the basis $\left\{A_{0}, U, Z_{j}, Z_{j}^{\prime}: 1 \leqslant j \leqslant n-1\right\}$ of $\mathfrak{a}+\mathfrak{n}$ and the generators of $Z_{\mathfrak{k}}(\mathfrak{a})$ above, we get the basis $\left\{C_{r}, F_{j k}, H_{j k}, U_{\mathfrak{k}},\left(Z_{r}\right)_{\mathfrak{k}},\left(Z_{r}^{\prime}\right)_{\mathfrak{k}}: r, j, k=1, \ldots, n-1, j<k\right\}$ of $\mathfrak{k}$, and the basis $\left\{A_{0}, U_{\mathfrak{p}},\left(Z_{j}\right)_{\mathfrak{p}},\left(Z_{j}^{\prime}\right)_{\mathfrak{p}}: 1 \leqslant j \leqslant n-1\right\}$ of $\mathfrak{p}$. Notice that, if $n=1, \mathfrak{k}=\left\langle U_{\mathfrak{k}}\right\rangle$ and $\mathfrak{p}=\left\langle A_{0}, U_{\mathfrak{p}}\right\rangle$, and if $n=2$ we have $\mathfrak{k}=\left\langle C, U_{\mathfrak{k}}, Z_{\mathfrak{k}}, Z_{\mathfrak{k}}^{\prime}\right\rangle$, and $\mathfrak{p}=\left\langle A, U_{\mathfrak{p}}, Z_{\mathfrak{p}}, Z_{\mathfrak{p}}^{\prime}\right\rangle$. We 
also decompose $\mathfrak{k}=\mathfrak{k}^{\prime}+\mathfrak{c}$, where $\mathfrak{k}^{\prime}=[\mathfrak{k}, \mathfrak{k}]=\left\langle C_{r}-U_{\mathfrak{k}}, F_{j k}, H_{j k},\left(Z_{r}\right)_{\mathfrak{k}},\left(Z_{r}^{\prime}\right)_{\mathfrak{k}}: r, j, k=\right.$ $1, \ldots, n-1, j<k\rangle \cong \mathfrak{s u}(n)$, and $\mathfrak{c}$ is the center of $\mathfrak{k}$, which is generated by the element $E_{J}=\frac{1}{2 n+1}\left(C_{1}+\cdots+C_{n-1}+(n+1) U_{\mathfrak{k}}\right)$ such that $\operatorname{ad}_{E_{J}}: \mathfrak{p} \rightarrow \mathfrak{p}$ defines the complex structure on $\mathbb{C H}(n)$. By the isomorphisms $\mathfrak{p} \cong \mathfrak{s u}(n, 1) / \mathfrak{k} \cong \mathfrak{a}+\mathfrak{n}$, we obtain the complex structure $J$ acting on $\mathfrak{a}+\mathfrak{n}$ as follows.

$$
J A_{0}=-U, \quad J U=A_{0}, \quad J Z_{r}=Z_{r}^{\prime}, \quad J Z_{r}^{\prime}=-Z_{r} .
$$

We consider the scalar product $\langle$,$\rangle on \mathfrak{a}+\mathfrak{n}$ defined by the isomorphism $\mathfrak{a}+\mathfrak{n} \cong \mathfrak{p}$ and $\frac{1}{4(n+1)} B_{\mid \mathfrak{p} \times \mathfrak{p}}$. Then $(\mathfrak{a}+\mathfrak{n},\langle\rangle, J$,$) is a Hermitian vector space, and the basis$ $\left\{A_{0}, U, Z_{r}, Z_{r}^{\prime}: 1 \leqslant r \leqslant n-1\right\}$ of $\mathfrak{a}+\mathfrak{n}$ is orthonormal. We consider the solvable factor $A N$ (with Lie algebra $\mathfrak{a}+\mathfrak{n}$ ) of the Iwasawa decomposition of $S U(n, 1)$ with the invariant metric $g$ and almost complex structure $J$ defined by $\langle$,$\rangle and J$, respectively.

The Lie brackets of the elements of the basis of $\mathfrak{a}+\mathfrak{n}$ are given by

$$
\begin{gathered}
{\left[A_{0}, U\right]=2 U, \quad\left[A_{0}, Z_{j}\right]=Z_{j}, \quad\left[A_{0}, Z_{j}^{\prime}\right]=Z_{j}^{\prime}, \quad\left[Z_{j}, Z_{r}^{\prime}\right]=-\delta_{j r} 2 U,} \\
{\left[U, Z_{j}\right]=\left[U, Z_{j}^{\prime}\right]=\left[Z_{j}, Z_{r}\right]=\left[Z_{j}^{\prime}, Z_{r}^{\prime}\right]=0 .}
\end{gathered}
$$

The Levi-Civita connection $\nabla$ is given by $2 g\left(\nabla_{X} Y, Z\right)=g([X, Y], Z)-g([Y, Z], X)+$ $g([Z, X], Y)$ for all $X, Y, Z \in \mathfrak{a}+\mathfrak{n}$. So, the covariant derivatives between generators of $\mathfrak{a}+\mathfrak{n}$ are given by

$$
\begin{gathered}
\nabla_{A_{0}} A_{0}=\nabla_{A_{0}} U=\nabla_{A_{0}} Z_{r}=\nabla_{A_{0}} Z_{r}^{\prime}=0 \\
\nabla_{U} A_{0}=-2 U, \quad \nabla_{U} U=2 A_{0}, \quad \nabla_{U} Z_{r}=Z_{r}^{\prime}, \quad \nabla_{U} Z_{r}^{\prime}=-Z_{r}, \\
\nabla_{Z_{j}} A_{0}=-Z_{j}, \quad \nabla_{Z_{j}} U=Z_{j}^{\prime}, \quad \nabla_{Z_{j}} Z_{r}=\delta_{j r} A_{0}, \quad \nabla_{Z_{j}} Z_{r}^{\prime}=-\delta_{j r} U, \\
\nabla_{Z_{j}^{\prime}} A_{0}=-Z_{j}^{\prime}, \quad \nabla_{Z_{j}^{\prime}} U=-Z_{j}, \quad \nabla_{Z_{j}^{\prime}} Z_{r}=\delta_{j r} U, \quad \nabla_{Z_{j}^{\prime}} Z_{r}^{\prime}=\delta_{j r} A_{0} .
\end{gathered}
$$

The components of the curvature tensor field $R$ are given by

$$
\begin{array}{llll}
R_{A_{0} U} A_{0}=-4 U, & R_{A_{0} U} U=4 A_{0}, & R_{A_{0} U} Z_{r}=2 Z_{r}^{\prime}, & R_{A_{0} U} Z_{r}^{\prime}=-2 Z_{r}, \\
R_{A_{0} Z_{j}} A_{0}=-Z_{j}, & R_{A_{0} Z_{j}} U=Z_{j}^{\prime}, & R_{A_{0} Z_{j}} Z_{r}=\delta_{j r} A_{0}, & R_{A_{0} Z_{j}} Z_{r}^{\prime}=-\delta_{j r} U, \\
R_{A_{0} Z_{j}^{\prime}} A_{0}=-Z_{j}^{\prime}, & R_{A_{0} Z_{j}^{\prime}} U=-Z_{j}, & R_{A_{0} Z_{j}^{\prime}} Z_{r}=\delta_{j r} U, & R_{A_{0} Z_{j}^{\prime}} Z_{r}^{\prime}=\delta_{j r} A_{0}, \\
R_{U Z_{j}} A_{0}=-Z_{j}^{\prime}, & R_{U Z_{j}} A_{0}=-Z_{j}, & R_{U Z_{j}} Z_{r}=\delta_{j r} U, & R_{U Z_{j}} Z_{r}^{\prime}=\delta_{j r} A_{0}, \\
R_{U Z_{j}^{\prime}} A_{0}=Z_{j}, \quad R_{U Z_{j}^{\prime}} U=-Z_{j}^{\prime}, & R_{U Z_{j}^{\prime}} Z_{r}=-\delta_{j r} A_{0}, & R_{U Z_{j}^{\prime}} Z_{r}^{\prime}=\delta_{j r} U, \\
R_{Z_{k} Z_{j}} A_{0}=R_{Z_{k} Z_{j}} U=0, & R_{Z_{j} Z_{r}^{\prime}} A_{0}=2 \delta_{j r} U, \quad R_{Z_{j} Z_{r}^{\prime}} U=-2 \delta_{j r} A_{0}, \\
R_{Z_{k} Z_{j}} Z_{r}=\delta_{j r} Z_{k}-\delta_{k r} Z_{j}, & R_{Z_{k} Z_{j}} Z_{r}^{\prime}=\delta_{j r} Z_{k}^{\prime}-\delta_{k r} Z_{j}^{\prime}, & R_{Z_{k}^{\prime} Z_{j}^{\prime}}=R_{Z_{k} Z_{j}}, \\
R_{Z_{j} Z_{j}^{\prime}} Z_{r}=-2\left(1+\delta_{j r} Z_{r}^{\prime}\right), & R_{Z_{j} Z_{j}} Z_{r}^{\prime}=2\left(1+\delta_{j r}\right) Z_{r} \\
R_{Z_{k} Z_{j}^{\prime}} Z_{r}=-\delta_{j r} Z_{k}^{\prime}-\delta_{k r} Z_{j}^{\prime}, & R_{Z_{k} Z_{j}^{\prime}} Z_{r}=\delta_{j r} Z_{k}-\delta_{k r} Z_{j}, \quad(k \neq j) .
\end{array}
$$

In particular we see that the invariant metric on $A N$ has constant holomorphic sectional curvature -4 .

\subsection{Homogeneous Kähler structures on $\mathbb{C H}(n) \equiv A N$}

We will determine the homogeneous Kähler structures on $\mathbb{C H}(n) \equiv A N$ in terms of the basis of left-invariant forms $\alpha, \beta, \gamma^{j}, \gamma^{\prime j}, 1 \leqslant j \leqslant n-1$, dual to $A_{0}, U, Z_{j}, Z_{j}^{\prime}$. If $S$ is 
a homogeneous Riemannian structure on $A N$ and $\widetilde{\nabla}=\nabla-S$, the condition $\widetilde{\nabla} g=0$ in (2.1) is equivalent to $S_{X Y Z}+S_{X Z Y}=0$ for all $X, Y, Z \in \mathfrak{a}+\mathfrak{n}$. Moreover, $\widetilde{\nabla} R=0$ is equivalent to the condition

$$
\left(\nabla_{X} R\right)_{Y_{1} Y_{2} Y_{3} Y_{4}}=-R_{S_{X} Y_{1} Y_{2} Y_{3} Y_{4}}-R_{Y_{1} S_{X} Y_{2} Y_{3} Y_{4}}-R_{Y_{1} Y_{2} S_{X} Y_{3} Y_{4}}-R_{Y_{1} Y_{2} Y_{3} S_{X} Y_{4}},
$$

for all $Y_{1}, Y_{1}, Y_{3}, Y_{4} \in \mathfrak{a}+\mathfrak{n}$. Replacing $\left(Y_{1}, Y_{2}, Y_{3}, Y_{4}\right)$ by $\left(A_{0}, U, A_{0}, Z_{j}\right),\left(A_{0}, U, A_{0}, Z_{j}^{\prime}\right)$, $\left(A_{0}, U, Z_{k}, Z_{j}\right)$, and $\left(A_{0}, U, Z_{k}, Z_{j}^{\prime}\right)$, one gets that $S_{X U Z_{j}}=S_{X A_{0} Z_{j}^{\prime}}, S_{X U Z_{j}^{\prime}}=-S_{X A_{0} Z_{j}}$, $S_{X Z_{k} Z_{j}^{\prime}}=-S_{X Z_{k}^{\prime} Z_{j}}$, and $S_{X Z_{k} Z_{j}}=S_{X Z_{k}^{\prime} Z_{j}^{\prime}}$, respectively. It is easy to see that the condition $\widetilde{\nabla} R=0$ holds if and only if the last four Eqs. are satisfied for all $X \in \mathfrak{a}+\mathfrak{n}$. These Eqs. also show (see (3.2)) that the condition $S \cdot J=0$ of homogeneous Kähler structure (see Proposition 2.2) is fulfilled. We put

$$
\begin{gathered}
\omega(X)=S_{X A_{0} U}, \quad \sigma^{j}(X)=S_{X A_{0} Z_{j}}=-S_{X U Z_{j}^{\prime}}, \quad \tau^{j}(X)=S_{X A_{0} Z_{j}^{\prime}}=S_{X U Z_{j}}, \\
\theta^{k j}(X)=S_{X Z_{k} Z_{j}^{\prime}}=S_{X Z_{j} Z_{k}^{\prime}}, \quad \psi^{k j}(X)=S_{X Z_{k} Z_{j}}=S_{X Z_{k}^{\prime} Z_{j}^{\prime}} .
\end{gathered}
$$

We have $\theta^{k j}=\theta^{j k}$ and $\psi^{k j}=-\psi^{j k}$. Now, we must determine the conditions for the 1-forms $\omega, \sigma^{j}, \tau^{j}, \theta^{k j}$ and $\sigma^{k j}$ under which the condition $\widetilde{\nabla} S=0$ in (2.1) is satisfied.

By (3.3), (3.4) and (3.5), the connection $\widetilde{\nabla}=\nabla-S$ is given by

$$
\begin{aligned}
\widetilde{\nabla}_{X} A_{0}= & -(2 \beta+\omega)(X) U-\sum_{j}\left(\gamma^{j}+\sigma^{j}\right)(X) Z_{j}-\sum_{j}\left(\gamma^{j}+\tau^{j}\right)(X) Z_{j}^{\prime}, \\
\widetilde{\nabla}_{X} U= & (2 \beta+\omega)(X) A_{0}-\sum_{j}\left(\gamma^{\prime j}+\tau^{j}\right)(X) Z_{j}+\sum_{j}\left(\gamma^{j}+\sigma^{j}\right)(X) Z_{j}^{\prime}, \\
\widetilde{\nabla}_{X} Z_{j}= & \left(\gamma^{j}+\sigma^{j}\right)(X) A_{0}+\left(\gamma^{\prime j}+\tau^{j}\right)(X) U+\left(\beta-\theta^{j}\right)(X) Z_{j}^{\prime} \\
& +\sum_{k \neq j}\left(\psi^{k j}(X) Z_{k}-\theta^{k j}(X) Z_{k}^{\prime}\right), \\
\widetilde{\nabla}_{X} Z_{j}^{\prime}= & \left(\gamma^{\prime j}+\tau^{j}\right)(X) A_{0}-\left(\gamma^{j}+\sigma^{j}\right)(X) U+\left(\theta^{j}-\beta\right)(X) Z_{j} \\
& +\sum_{k \neq j}\left(\theta^{k j}(X) Z_{k}-\psi^{k j}(X) Z_{k}^{\prime}\right) .
\end{aligned}
$$

Now, replacing $\left(V_{1}, V_{2}\right)$ in the Eq. $\left(\widetilde{\nabla}_{X} S\right)\left(W, V_{1}, V_{2}\right)=0$ by $\left(A_{0}, U\right),\left(A_{0}, Z_{j}\right),\left(A_{0}, Z_{j}^{\prime}\right)$, $\left(Z_{k}, Z_{j}\right)$ and $\left(Z_{k}, Z_{j}^{\prime}\right)$, respectively, we obtain that the condition $\widetilde{\nabla} S=0$ is equivalent to the following conditions:

$$
\begin{aligned}
\widetilde{\nabla} \omega & =2 \sum_{j}\left(\left(\gamma^{j}+\sigma^{j}\right) \otimes \tau^{j}-\left(\gamma^{\prime j}+\tau^{j}\right) \otimes \sigma^{j}\right), \\
\widetilde{\nabla} \sigma^{j} & =-\left(\beta+\omega+\theta^{j}\right) \otimes \tau^{j}+\left(\gamma^{\prime j}+\tau^{j}\right) \otimes\left(\omega+\theta^{j}\right) \\
& +\sum_{k \neq j}\left(\psi^{k j} \otimes \sigma^{k}-\theta^{k j} \otimes \tau^{k}+\left(\gamma^{\prime k}+\tau^{k}\right) \otimes \theta^{k j}-\left(\gamma^{k}+\sigma^{k}\right) \otimes \psi^{k j}\right), \\
\widetilde{\nabla} \tau^{j} & =\left(\beta+\omega+\theta^{j}\right) \otimes \sigma^{j}-\left(\gamma^{j}+\sigma^{j}\right) \otimes\left(\omega+\theta^{j}\right) \\
& +\sum_{k \neq j}\left(\theta^{k j} \otimes \sigma^{k}+\psi^{k j} \otimes \tau^{k}-\left(\gamma^{k}+\sigma^{k}\right) \otimes \theta^{k j}-\left(\gamma^{\prime k}+\tau^{k}\right) \otimes \psi^{k j}\right), \\
\widetilde{\nabla} \theta^{k j} & =\left(\gamma^{j}+\sigma^{j}\right) \otimes \tau^{k}+\left(\gamma^{k}+\tau^{k}\right) \otimes \tau^{j}-\left(\gamma^{\prime j}+\tau^{j}\right) \otimes \sigma^{k}-\left(\gamma^{\prime k}+\tau^{k}\right) \otimes \sigma^{j} \\
& +\sum_{l} \psi^{l k} \wedge \theta^{j l}+\sum_{l} \theta^{l k} \wedge \psi^{j l}, \\
\widetilde{\nabla} \psi^{k j} & =\left(\gamma^{k}+\sigma^{k}\right) \otimes \sigma^{j}-\left(\gamma^{j}+\sigma^{j}\right) \otimes \sigma^{k}-\left(\gamma^{\prime k}+\tau^{k}\right) \otimes \tau^{j}-\left(\gamma^{\prime j}+\tau^{j}\right) \otimes \tau^{k} \\
& +\sum_{l} \theta^{l k} \wedge \theta^{j l}-\sum \psi^{l k} \wedge \psi^{j l},
\end{aligned}
$$

where $\theta^{j}=\theta^{j j}$. Thus, from (3.4) and (3.5), we have 
Theorem 3.1. All the homogeneous Kähler structures on $\mathbb{C H}(n) \equiv A N$ are given by

$$
\begin{aligned}
S=\omega \otimes(\alpha \wedge \beta) & +\sum_{j=1}^{n-1}\left(\sigma^{j} \otimes\left(\alpha \wedge \gamma^{j}-\beta \wedge \gamma^{j}\right)+\tau^{j} \otimes\left(\alpha \wedge \gamma^{j}+\beta \wedge \gamma^{j}\right)+\theta^{j j} \otimes\left(\gamma^{j} \wedge \gamma^{\prime j}\right)\right) \\
& +\sum_{1 \leqslant k<j \leqslant n-1}\left(\psi^{k j} \otimes\left(\gamma^{k} \wedge \gamma^{j}+\gamma^{\prime k} \wedge \gamma^{\prime j}\right)+\theta^{k j} \otimes\left(\gamma^{k} \wedge \gamma^{\prime j}+\gamma^{j} \wedge \gamma^{\prime k}\right)\right),
\end{aligned}
$$

where $\omega, \sigma^{j}, \tau^{j}, \theta^{k j}, \psi^{k j},(1 \leqslant k, j \leqslant n-1)$, are 1 -forms on AN satisfying $\theta^{j k}=\theta^{k j}$, $\psi^{j k}=-\psi^{k j}$ and the Eqs. (3.6).

If $n=2$, we put $\gamma=\gamma^{1}, \gamma^{\prime}=\gamma^{\prime 1}$, so that $\left\{\alpha, \beta, \gamma, \gamma^{\prime}\right\}$ is the basis of left-invariant forms on $A N=\mathbb{C H}(2)$ dual to $\left\{A_{0}, U, Z, Z^{\prime}\right\}$, and we have

Corollary 3.2. All the homogeneous Kähler structures on the complex hyperbolic plane $\mathbb{C H}(2) \equiv A N$ are given by

$$
S=\omega \otimes(\alpha \wedge \beta)+\sigma \otimes\left(\alpha \wedge \gamma-\beta \wedge \gamma^{\prime}\right)+\tau \otimes\left(\alpha \wedge \gamma^{\prime}+\beta \wedge \gamma\right)+\theta \otimes\left(\gamma \wedge \gamma^{\prime}\right),
$$

where $\omega, \sigma, \tau$ and $\theta$ are 1 -forms on AN satisfying

$$
\begin{aligned}
& \widetilde{\nabla} \omega=2(\gamma+\sigma) \otimes \tau-2\left(\gamma^{\prime}+\tau\right) \otimes \sigma=\widetilde{\nabla} \theta, \\
& \widetilde{\nabla} \sigma=-(\beta+\omega+\theta) \otimes \gamma+\left(\gamma^{\prime}+\tau\right) \otimes(\omega+\theta), \\
& \widetilde{\nabla} \tau=(\beta+\omega+\theta) \otimes \sigma-(\gamma+\sigma) \otimes(\omega+\theta) .
\end{aligned}
$$

If $n=1,\{\alpha, \beta\}$ is the basis of 1-invariant forms on the 2-dimensional solvable Lie group $A N=\mathbb{C H}(1)$ dual to the basis $\left\{A_{0}, U\right\}$ of $\mathfrak{a}+\mathfrak{n}$, and we have

Corollary 3.3. All the homogeneous Kähler structures on the complex hyperbolic line (or real hyperbolic plane) $\mathbb{C H}(1) \equiv A N$ are given by $S=\omega \otimes(\alpha \wedge \beta)$, where $\omega$ is a 1-form on $A N$ satisfying $\widetilde{\nabla} \omega=0$.

Remark 3.4. If $S=\omega \otimes(\alpha \wedge \beta)$ is a homogeneous Kähler structure on $\mathbb{C H}(1)$, and $\omega=\lambda \alpha+\mu \beta$, where $\lambda$ and $\mu$ are functions on $\mathbb{C H}(1)$, the condition $\widetilde{\nabla} \omega=0$ together with the structure Eq. $\left[A_{0}, U\right]=2 U$ gives $\lambda=\mu=0$ or $\lambda^{2}+\mu^{2}=4$, and we have that there are infinite homogeneous Kähler structures on $\mathbb{C H}(1)$. However (see [26], Th. 4.4), up to isomorphism, there are only two homogeneous structures on the real hyperbolic plane: one of them is $S=0(\lambda=\mu=0)$, and the other, which is given by $S_{X} Y=g(X, Y) \xi_{0}-g\left(\xi_{0}, Y\right) X$, with $\xi_{0}=2 A_{0}$ (for $X, Y \in \mathfrak{a}+\mathfrak{n}=\left\langle A_{0}, U\right\rangle$ ), corresponds to $S=\omega \otimes(\alpha \wedge \beta)$, with $\omega=-2 \beta(\lambda=0, \mu=-2)$.

Remark 3.5. For each $n>0, S=0$ is a homogeneous Kähler structure on $\mathbb{C H}(n) \equiv$ $A N$, the corresponding canonical connection is $\widetilde{\nabla}=\nabla$, its holonomy algebra is $\mathfrak{k} \cong$ $\mathfrak{s}(\mathfrak{u}(n) \oplus \mathfrak{u}(1))$, the associated reductive decomposition is the Cartan decomposition $\mathfrak{s u}(n, 1)=\mathfrak{k}+\mathfrak{p}$, and it gives the description of $\mathbb{C H}(n)$ as symmetric space $\mathbb{C H}(n)=$ $S U(n, 1) / S(U(n) \times U(1))$. 
Now, our purpose is to obtain nontrivial homogeneous Kähler structures on $\mathbb{C H}(n)$, $n \geqslant 2$, their associated reductive decompositions, and the corresponding descriptions as homogeneous Kähler spaces.

We will seek for solutions for which $\sigma^{j}=-\gamma^{j}, \tau^{j}=-\gamma^{\prime j}$. In this case, we have

$$
\begin{aligned}
\widetilde{\nabla} \gamma^{j} & =\left(\beta-\theta^{j}\right) \otimes \gamma^{\prime j}+\sum_{k \neq j}\left(\psi^{k j} \otimes \gamma^{k}-\theta^{k j} \otimes \gamma^{\prime k}\right), \\
\widetilde{\nabla} \gamma^{\prime j} & =\left(\theta^{j}-\beta\right) \otimes \gamma^{j}+\sum_{k \neq j}\left(\theta^{k j} \otimes \gamma^{k}+\psi^{k j} \otimes \gamma^{\prime k}\right) .
\end{aligned}
$$

(Obviously, the last summands on the right hand-side in each one of the two Eqs. above do not appear if $n=2$.) By the second and third Eqs. in (3.6), we must have $\omega=-2 \beta$, which also satisfies the first Eqs. in (3.6), because $\widetilde{\nabla} \beta=(2 \beta+\omega) \otimes \alpha-\sum_{j}\left(\gamma^{\prime j}+\tau^{j}\right) \otimes$ $\gamma^{j}+\sum_{j}\left(\gamma^{j}+\sigma^{j}\right) \otimes \gamma^{j}=0$. If $n=2$, by Corollary 3.2, we only have to determine $\theta$ such that $\widetilde{\nabla} \theta=0$. If we put $\theta=a \alpha+b \beta+c \gamma+c^{\prime} \gamma^{\prime}$, by using also the structure Eqs. of $\mathfrak{a}+\mathfrak{n}=\left\langle A_{0}, U, Z, Z^{\prime}\right\rangle$, we obtain that $c=c^{\prime}=0$ and $a$ and $b$ are constant. For $n>2$ we put $\theta^{j}=\theta^{j j}=a_{j} \alpha+b_{j} \beta, \theta^{k j}=c_{k j} \alpha, \psi^{k j}=p_{k j} \alpha,(k \neq j)$, with $a_{j}, b_{j}, c_{k j}, p_{k j} \in \mathbb{R}$. Then, if $\sigma^{j}=-\gamma^{j}, \tau^{j}=-\gamma^{\prime j}$ and $\omega=-2 \beta$, Eqs. (3.6) are satisfied if and only if one has

$$
p_{k j}\left(b_{k}-b_{j}\right)=c_{k j}\left(b_{k}-b_{j}\right)=0 .
$$

Consequently, we get

Proposition 3.6. For $n>2$, the space $\mathbb{C H}(n)$ admits the multi-parametric family of

\begin{tabular}{|c|c|c|c|c|}
\hline Table I & $A_{0}$ & $U$ & $Z_{j}$ & $Z_{j}^{\prime}$ \\
\hline$S_{A_{0}}$ & 0 & 0 & $a_{j} Z_{j}^{\prime}+\sum_{l \neq j}\left(p_{j l} Z_{l}+c_{j l} Z_{l}^{\prime}\right)$ & $-a_{j} Z_{j}+\sum_{l \neq j}\left(p_{j l} Z_{l}^{\prime}-c_{j l} Z_{l}\right)$ \\
\hline$S_{U}$ & $-2 U$ & $2 A_{0}$ & $b_{j} Z_{j}^{\prime}$ & $-b_{j} Z_{j}$ \\
\hline$S_{Z_{k}}$ & $-Z_{k}$ & $Z_{k}^{\prime}$ & $\delta_{k j} A_{0}$ & $-\delta_{k j} U$ \\
\hline$S_{Z_{k}^{\prime}}$ & $-Z_{k}^{\prime}$ & $-Z_{k}$ & $\delta_{k j} U$ & $\delta_{k j} A_{0}$ \\
\hline
\end{tabular}
homogeneous Kähler structures $S=S^{a_{j}, b_{j}, c_{k j}, p_{k j}}$ given in terms of the generators of $\mathfrak{a}+\mathfrak{n}$ by the following table.

\begin{tabular}{|c|c|c|c|c|}
\hline Table II & $A_{0}$ & $U$ & $Z$ & $Z^{\prime}$ \\
\hline$S_{A_{0}}$ & 0 & 0 & $a Z^{\prime}$ & $-a Z$ \\
\hline$S_{U}$ & $-2 U$ & $2 A_{0}$ & $b Z^{\prime}$ & $-b Z$ \\
\hline$S_{Z}$ & $-Z$ & $Z^{\prime}$ & $A_{0}$ & $-U$ \\
\hline$S_{Z^{\prime}}$ & $-Z^{\prime}$ & $-Z$ & $U$ & $A_{0}$ \\
\hline
\end{tabular}

The complex hyperbolic plane $\mathbb{C H}(2)$ admits the two-parametric family of homogeneous Kähler structures $S=S^{a, b}$ given in terms of the generators of $\mathfrak{a}+\mathfrak{n}$ by the following table.

If $S=S^{a_{j}, b_{j}, c_{k j}, p_{k j}}$, with respect to the basis $\left\{A_{0}, U, Z_{j}, Z_{j}^{\prime}\right\}$ of $\mathfrak{a}+\mathfrak{n}$, the connection $\widetilde{\nabla}=\nabla-S$ is given by

$$
\begin{array}{cc}
\widetilde{\nabla}_{A_{0}} Z_{j}=-a_{j} Z_{j}^{\prime}-\sum_{l \neq j}\left(p_{j l} Z_{l}+c_{j l} Z_{l}^{\prime}\right), & \widetilde{\nabla}_{U} Z_{j}=\left(1-b_{j}\right) Z_{j}^{\prime} \\
\widetilde{\nabla}_{A_{0}} Z_{j}^{\prime}=a_{j} Z_{j}-\sum_{l \neq j}\left(p_{j l} Z_{l}^{\prime}-c_{j l} Z_{l}\right), & \widetilde{\nabla}_{U} Z_{j}^{\prime}=\left(b_{j}-1\right) Z_{j}
\end{array}
$$


with the rest vanishing. Hence, the components of the curvature tensor field are $\widetilde{R}_{A_{0} U}=$ $-\widetilde{R}_{Z_{k} Z_{k}^{\prime}}=2 \sum_{j}\left(1-b_{j}\right)\left(Z_{j}^{\prime} \otimes \gamma^{j}-Z_{j} \otimes \gamma^{\prime j}\right)$, and the rest zero.

If $b_{j}=1$ for all $j=1, \ldots, n-1$, the holonomy algebra of $\widetilde{\nabla}$ is trivial and the reductive decompositions associated to the homogeneous Kähler structures given in Proposition 3.6 are given by $\widetilde{\mathfrak{g}}^{a_{j}, c_{k j}, p_{k j}}=\{0\}+(\mathfrak{a}+\mathfrak{n})$ with nonvanishing brackets, by (2.3), given by

$$
\begin{aligned}
& {\left[A_{0}, U\right]=2 U, \quad\left[A_{0}, Z_{j}\right]=Z_{j}+a_{j} Z_{j}^{\prime}+\sum_{l \neq j}\left(p_{j l} Z_{l}+c_{j l} Z_{l}^{\prime}\right),} \\
& {\left[A_{0}, Z_{j}^{\prime}\right]=-a_{j} Z_{j}+Z_{j}^{\prime}+\sum_{l \neq j}\left(p_{j l} Z_{l}^{\prime}+c_{j l} Z_{l}\right), \quad\left[Z_{j}, Z_{j}^{\prime}\right]=-2 U .}
\end{aligned}
$$

On the other hand, the element $\hat{A}_{0}=\lambda_{1} C_{1}+\cdots+\lambda_{n-1} C_{n-1}+\sum_{j<l}\left(c_{j l} H_{j l}-p_{j l} F_{j l}\right)+A_{0}$ of $\mathfrak{s u}(n, 1)$ generates a subspace $\mathfrak{e}^{\lambda_{j}, c_{k j}, p_{k j}}$ of $Z_{\mathfrak{k}}(\mathfrak{a})+\mathfrak{a}$, and the structure Eqs. of the Lie subalgebra $\mathfrak{e}^{\lambda_{j}, c_{k j}, p_{k j}}+\mathfrak{n}$ of $\mathfrak{s u}(n, 1)$ are

$$
\begin{aligned}
& {\left[\hat{A}_{0}, U\right]=2 U, \quad\left[\hat{A}_{0}, Z_{j}\right]=Z_{j}+\left(3 \lambda_{j}+\sum_{l \neq j} \lambda_{l}\right) Z_{j}^{\prime}+\sum_{l \neq j}\left(p_{j l} Z_{l}+c_{j l} Z_{l}^{\prime}\right),} \\
& {\left[\hat{A}_{0}, Z_{j}^{\prime}\right]=-\left(3 \lambda_{j}+\sum_{l \neq j} \lambda_{l}\right) Z_{j}+Z_{j}^{\prime}+\sum_{l \neq j}\left(p_{j l} Z_{l}^{\prime}+c_{j l} Z_{l}\right), \quad\left[Z_{j}, Z_{j}^{\prime}\right]=-2 U,}
\end{aligned}
$$

with the rest vanishing. From (3.7) and (3.8), it follows that $\widetilde{\mathfrak{g}}^{a_{j}, c_{k j}, p_{k j}}$ is isomorphic to $\mathfrak{e}^{\lambda_{j}, c_{k j}, p_{k j}}+\mathfrak{n}$.

Now, for the structure $S=S^{a_{j}, b_{j}, c_{k j}, p_{k j}}$ in Table I, suppose that $b_{j} \neq 1$ for some $j=$ $1, \ldots, n-1$. Then, $\rho=\widetilde{R}_{A_{0} U}=-\widetilde{R}_{Z_{k} Z_{k}^{\prime}}=2 \sum_{j}\left(1-b_{j}\right)\left(Z_{j}^{\prime} \otimes \gamma^{j}-Z_{j} \otimes \gamma^{\prime j}\right)$ generates the holonomy algebra $\widetilde{\mathfrak{h}}^{a_{j}, b_{j}, c_{k j}, p_{k j}}$ of $\widetilde{\nabla}=\nabla-S$, and the reductive decomposition associated to $S$ is $\widetilde{\mathfrak{g}}^{a_{j}, b_{j}, c_{k j}, p_{k j}}=\widetilde{\mathfrak{h}}^{a_{j}, b_{j}, c_{k j}, p_{k j}}+(\mathfrak{a}+\mathfrak{n})=\left\langle\rho, A_{0}, U, Z_{j}, Z_{j}^{\prime}\right\rangle$ with structure Eqs., by (2.3), given by

$$
\begin{aligned}
& {\left[\rho, A_{0}\right]=[\rho, U]=0, \quad\left[\rho, Z_{j}\right]=2\left(1-b_{j}\right) Z_{j}^{\prime}, \quad\left[\rho, Z_{j}^{\prime}\right]=2\left(b_{j}-1\right) Z_{j},} \\
& {\left[A_{0}, U\right]=\rho+2 U, \quad\left[A_{0}, Z_{j}\right]=Z_{j}+a_{j} Z_{j}^{\prime}+\sum_{l \neq j}\left(p_{j l} Z_{l}+c_{j l} Z_{l}^{\prime}\right),} \\
& {\left[A_{0}, Z_{j}^{\prime}\right]=-a_{j} Z_{j}+Z_{j}^{\prime}+\sum_{l \neq j}\left(p_{j l} Z_{l}^{\prime}+c_{j l} Z_{l}\right),} \\
& {\left[U, Z_{j}\right]=\left(b_{j}-1\right) Z_{j}^{\prime}, \quad\left[U, Z_{j}^{\prime}\right]=\left(1-b_{j}\right) Z_{j}, \quad\left[Z_{k}, Z_{j}^{\prime}\right]=-\delta_{k j}(\rho+2 U) .}
\end{aligned}
$$

If $\mathfrak{u} \cong \mathfrak{u}(1)$ is the subspace of $Z_{\mathfrak{k}}(\mathfrak{a})$ generated by $C=C_{1}+\cdots+C_{n-1}$, it is easy to see that the Lie algebra $\widetilde{\mathfrak{g}}^{a_{j}, b_{j}, c_{k j}, p_{k j}}$ is isomorphic to the Lie subalgebra $\mathfrak{u}+\mathfrak{e}^{\lambda_{j}, c_{k j}, p_{k j}}+\mathfrak{n}=$ $\left\langle C, \hat{A}_{0}, U, Z_{j}, Z_{j}^{\prime}\right\rangle$ of $\mathfrak{s u}(n, 1)$. We deduce

Theorem 3.7. Let $S=S^{a_{j}, b_{j}, c_{k j}, p_{k j}}$ be the homogeneous Kähler structure on $\mathbb{C H}(n)$, $n>2$, given by Table I, and let $\mathfrak{e}^{\lambda_{j}, c_{k j}, p_{k j}}$ be the subspace of $Z_{\mathfrak{k}}(\mathfrak{a})+\mathfrak{a}$ generated by

$$
\hat{A}_{0}=\sum_{j} \lambda_{j} C_{j}+\sum_{1 \leqslant j<l \leqslant n-1}\left(c_{j l} H_{j l}-p_{j l} F_{j l}\right)+A_{0}, \quad\left(\lambda_{j}=\frac{n a_{j}-\sum_{l \neq j} a_{l}}{2 n+2}\right),
$$

and $\mathfrak{u}=\left\langle C_{1}+\cdots+C_{n-1}\right\rangle$. If $b_{j}=1$ for all $j=1, \ldots, n-1$, the corresponding group of isometries is the connected subgroup $E^{\lambda_{j}, c_{k j}, p_{k j}} N$ of $S U(n, 1)$ whose lie algebra is $\mathfrak{e}^{\lambda_{j}, c_{k j}, p_{k j}}+\mathfrak{n}$. If $b_{j} \neq 1$ for some $j=1, \ldots, n-1$, the corresponding group of isometries is the connected subgroup $U(1) E^{\lambda_{j}, c_{k_{j}}, p_{k_{j}}} N$ of $S U(n, 1)$ whose Lie algebra is $\mathfrak{u}+\mathfrak{e}^{\lambda_{j}, c_{k j}, p_{k j}}+\mathfrak{n}$. 
If $S^{a, b}$ is the homogeneous Kähler structure on the complex hyperbolic plane $\mathbb{C H}(2)$ given by Table II, $\mathfrak{e}^{\lambda}=\left\langle\hat{A}_{0}\right\rangle$, where $\hat{A}_{0}=\lambda C+A_{0},(\lambda=a / 3)$, and $\mathfrak{u}=\langle C\rangle$, then the corresponding group of isometries is (i) the subgroup $E^{\lambda} N$ of $S U(2,1)$ generated by the Lie subalgebra $\mathfrak{e}^{\lambda}+\mathfrak{n}$ of $\mathfrak{s u}(2,1)$, if $b=1$; (ii) the subgroup $U(1) E^{\lambda} N$ of $S U(2,1)$ generated by $\mathfrak{u}+\mathfrak{e}^{\lambda}+\mathfrak{n}$, if $b \neq 1$.

Remark 3.8. Each structure $S^{a_{j}, b_{j}, c_{k j}, p_{k j}}$, with $b_{j}=1$ for all $j$, is also characterized by the fact that $\widetilde{\nabla}=\nabla-S^{a_{j}, b_{j}, c_{k j}, p_{k j}}$ is the canonical connection for the Lie group $E^{\lambda_{j}, c_{k j}, p_{k j}} N$, which is the connection for which every left-invariant vector field on $E^{\lambda_{j}, c_{k j}, p_{k j}} N$ is parallel. Each one of these groups acts simply transitively on $\mathbb{C H}(n)$ and it provides a description of $\mathbb{C H}(n)$ as a homogeneous space. If all the parameters $a_{j}, c_{k j}, p_{k j}$ are zero, then $\mathfrak{e}^{\lambda_{j}, c_{k j}, p_{k j}}=\mathfrak{a}$, and we get the usual description as a solvable Lie group $\mathbb{C H}(n)=A N$. In this case, the corresponding homogeneous structure is given by $S_{X} Y=\nabla_{X} Y$ for all $X, Y \in \mathfrak{a}+\mathfrak{n}$. If $b_{j} \neq 1$ for some $j=1, \ldots, n-1$, we get the descriptions as homogeneous space $\mathbb{C H}(n)=U(1) E^{\lambda_{j}, c_{k j}, p_{k j}} N / U(1)$.

\subsection{Principal line bundle over $\mathbb{C H}(n)$}

By (3.2), the fundamental 2-form of the Kähler structure $(J, g)$ of $\mathbb{C H}(n) \equiv A N$ is given by $\Omega=\alpha \wedge \beta-\sum_{j=1}^{n-1} \gamma^{j} \wedge \gamma^{\prime j}=-\frac{1}{2} d \beta$, where $\left\{\alpha, \beta, \gamma^{j}, \gamma^{\prime j}: 1 \leqslant j \leqslant n-1\right\}$ is the basis of left-invariant 1-forms on $A N$ dual to the basis $\left\{A_{0}, U, Z_{j}, Z_{j}^{\prime}\right\}$ of $\mathfrak{a}+\mathfrak{n}$. We consider the principal line bundle $\pi: \bar{M} \rightarrow \mathbb{C H}(n)$, and identify the bundle space $\bar{M}$ with $A N \times \mathbb{R}$ and $\pi$ with the projection on $A N$. The fundamental vector field $\xi$ is identified with $\frac{d}{d t}$, and the 1-form $\eta=d t-\pi^{*} \beta$ is also regarded as a connection form on the bundle. If $\varphi$ and $\bar{g}$ are given by $(2.7)$, then $(\varphi, \xi, \eta, \bar{g})$ is a Sasakian structure on $\bar{M}$.

By (a) of Proposition 2.5, each homogeneous Kähler structure $S^{a_{j}, b_{j}, c_{k j}, p_{k j}}$ on $\mathbb{C H}(n)$ given in Theorem 3.7 defines a homogeneous Sasakian structure $\bar{S}^{a_{j}, b_{j}, c_{k j}, p_{k j}}$ on $\bar{M}$ which gives a description of $\bar{M}$ as either the connected subgroup $E^{\lambda_{j}, c_{k j}, p_{k j}} N \times \mathbb{R}$ of $S U(n, 1) \times$ $\mathbb{R}$ (if $b_{j}=1$ for all $\left.j=1, \ldots, n-1\right)$, or as the homogeneous space $\left(U(1) E^{\lambda_{j}, c_{k j}, p_{k j}} N \times\right.$ $\mathbb{R}) / U(1)$.

On the other hand, from (b) of Proposition 2.5, it follows

Proposition 3.9. The bundle space $\bar{M}$ of the line bundle $\pi: \bar{M} \rightarrow \mathbb{C H}(n)$ admits the family of homogeneous Sasakian structures $\left\{S^{t}: t \in \mathbb{R}\right\}$ given, in terms of the horizontal lifts of the generators of $\mathfrak{a}+\mathfrak{n}$ and the fundamental vector field $\xi$, by the following table.

\begin{tabular}{|c|c|c|c|c|c|}
\hline Table III & $A_{0}^{H}$ & $U^{H}$ & $Z_{j}^{H}$ & $Z_{j}^{\prime H}$ & $\xi$ \\
\hline$S_{A_{0}^{H}}^{t}$ & 0 & $-\xi$ & 0 & 0 & $U^{H}$ \\
\hline$S_{U^{H}}^{t}$ & $\xi$ & 0 & 0 & 0 & $-A^{H}$ \\
\hline$S_{Z_{k}^{H}}^{t}$ & 0 & 0 & 0 & $\delta_{k j} \xi$ & $-Z_{k}^{\prime H}$ \\
\hline$S_{Z_{k}^{\prime H}}^{t}$ & 0 & 0 & $-\delta_{k j} \xi$ & 0 & $Z_{k}^{H}$ \\
\hline$S_{\xi}^{t}$ & $t U^{H}$ & $-t A^{H}$ & $-t Z_{j}^{\prime H}$ & $t Z_{j}^{H}$ & 0 \\
\hline
\end{tabular}

Remark 3.10. For each $p \in \bar{M}$, if $c_{12}\left(S^{t}\right)_{p}$ is the map from the tangent space $T_{p}(\bar{M})$ to its dual given by $c_{12}\left(S^{t}\right)_{p}(\tilde{X})=\sum_{i=1}^{2 n+1} S_{e_{i} e_{i} \tilde{X}}$, where $\left\{e_{i}\right\}$ is an orthonormal basis 
of $T_{p}(\bar{M})$, then $c_{12}\left(S^{t}\right)_{p}$ vanishes for every $t \in \mathbb{R}$. According to Tricerri-Vanhecke's classification of homogeneous Riemannian structures in [26], each $S^{t}$ is of type $\mathcal{T}_{2} \oplus \mathcal{T}_{3}$. Moreover, if $t=-1$, we have $S_{\tilde{X}} \tilde{Y}+S_{\tilde{Y}} \tilde{X}=0$, then $S^{-1}$ is of type $\mathcal{T}_{3}$, which means that $\bar{M}$ is a naturally reductive Riemannian space. If $t=2$, then each cyclic sum $\mathfrak{S}_{\tilde{X} \tilde{Y} \tilde{Z}} S_{\tilde{X} \tilde{Y} \tilde{Z}}$ vanishes, and hence $\bar{M}$ is of type $\mathcal{T}_{2}$, which may be also expressed by saying that $\bar{M}$ is a cotorsionless manifold (see [13]).

We will construct the reductive decomposition $\tilde{\mathfrak{g}}_{t}=\tilde{\mathfrak{h}}_{t}+\overline{\mathfrak{m}}$ associated to each homogeneous Sasakian structure $S^{t}$, where $\overline{\mathfrak{m}}=T_{o}(\bar{M})$, with $o \in \bar{M}$, is generated by $\tilde{A}=\left(A_{0}^{H}\right)_{o}$, $\tilde{U}=\left(U^{H}\right)_{o}, \tilde{Z}_{j}=\left(Z_{j}^{H}\right)_{o}, \tilde{Z}_{j}^{\prime}=\left(Z_{j}^{\prime}\right)_{o}^{H}, \bar{\xi}=\xi_{o},(1 \leqslant j \leqslant n-1)$, and $\tilde{\mathfrak{h}}_{t}$ is the holonomy algebra of the connection $\widetilde{D}^{t}=D-S^{t}$. Each connection $\widetilde{D}^{t}$ is given by

\begin{tabular}{|c|c|c|c|c|c|}
\hline Table IV & $A_{0}^{H}$ & $U^{H}$ & $Z_{j}^{H}$ & $Z_{j}^{\prime H}$ & $\xi$ \\
\hline$\widetilde{D}_{A_{0}^{H}}^{t}$ & 0 & 0 & 0 & 0 & 0 \\
\hline$\widetilde{D}_{U^{H}}^{t}$ & $-2 U^{H}$ & $2 A_{0}^{H}$ & $Z_{j}^{\prime H}$ & $-Z_{j}^{H}$ & 0 \\
\hline$\widetilde{D}_{Z_{k}^{H}}^{t}$ & $-Z_{k}^{H}$ & $Z_{k}^{\prime H}$ & $\delta_{k j} A_{0}^{H}$ & $-\delta_{k j} U^{H}$ & 0 \\
\hline$\widetilde{D}_{Z_{k}^{\prime H}}^{t^{k}}$ & $-Z_{k}^{\prime H}$ & $-Z_{k}^{H}$ & $\delta_{k j} U^{H}$ & $\delta_{k j} A_{0}^{H}$ & 0 \\
\hline$\widetilde{D}_{\xi}^{t}$ & $(1-t) U^{H}$ & $(t-1) A^{H}$ & $(t-1) Z_{j}^{\prime H}$ & $(1-t) Z_{j}^{H}$ & 0 \\
\hline
\end{tabular}

Let $\widetilde{R}^{t}$ be the curvature of $\widetilde{D}^{t}$, and let $\left\{\bar{\alpha}, \bar{\beta}, \bar{\gamma}^{j}, \bar{\gamma}^{\prime j}, \bar{\eta}\right\}$ be the basis dual to the basis $\left\{\tilde{A}, \tilde{U}, \tilde{Z}_{j}, \tilde{Z}_{j}^{\prime}, \bar{\xi}\right\}$ of $\overline{\mathfrak{m}}$. The holonomy algebra $\tilde{\mathfrak{h}}_{t}$ of $\widetilde{D}^{t}$ is generated by the curvature operators $\rho_{0}, \rho_{r}, \varphi_{r}, \psi_{r}, \sigma_{j k}, \tau_{j k}(r, j, k=1, \ldots, n-1, j<k)$, given by

$$
\begin{aligned}
\rho_{0}= & \widetilde{R}_{\tilde{A} \tilde{U}}^{t}=2(t-3)(\bar{\alpha} \otimes \tilde{U}-\bar{\beta} \otimes \tilde{A})+2(2-t) \sum_{j=1}^{n-1}\left(\bar{\gamma}^{j} \otimes \tilde{Z}_{j}^{\prime}-\bar{\gamma}^{\prime j} \otimes \tilde{Z}_{j}\right), \\
\rho_{r}= & \widetilde{R}_{\tilde{Z}_{r} \tilde{Z}_{r}^{\prime}}^{t}=2(2-t)(\bar{\alpha} \otimes \tilde{U}-\bar{\beta} \otimes \tilde{A})+2(t-3)\left(\bar{\gamma}^{r} \otimes \tilde{Z}_{r}^{\prime}-\bar{\gamma}^{\prime r} \otimes \tilde{Z}_{r}\right) \\
& +2(t-2) \sum_{j \neq r}\left(\bar{\gamma}^{j} \otimes \tilde{Z}_{j}^{\prime}-\bar{\gamma}^{\prime j} \otimes \tilde{Z}_{j}\right), \\
\varphi_{r}= & \widetilde{R}_{\tilde{A} \tilde{Z}_{r}}^{t}=-\widetilde{R}_{\tilde{U} \tilde{Z}_{r}^{\prime}}^{t}=-\bar{\alpha} \otimes \tilde{Z}_{r}+\bar{\beta} \otimes \tilde{Z}_{r}^{\prime}+\bar{\gamma}^{r} \otimes \tilde{A}-\bar{\gamma}^{\prime r} \otimes \tilde{U}, \\
\psi_{r}= & \widetilde{R}_{\tilde{U} \tilde{Z}_{r}}^{t}=\widetilde{R}_{\tilde{A} \tilde{Z}_{r}^{\prime}}^{t}=-\bar{\alpha} \otimes \tilde{Z}_{r}^{\prime}-\bar{\beta} \otimes \tilde{Z}_{r}+\bar{\gamma}^{r} \otimes \tilde{U}+\bar{\gamma}^{\prime r} \otimes \tilde{A}, \\
\sigma_{j k}= & \widetilde{R}_{\tilde{Z}_{j} \tilde{Z}_{k}}^{t}=\widetilde{R}_{\tilde{Z}_{j}^{\prime} \tilde{Z}_{k}^{\prime}}=-\bar{\gamma}^{j} \otimes \tilde{Z}_{k}-\bar{\gamma}^{\prime j} \otimes \tilde{Z}_{k}^{\prime}+\bar{\gamma}^{k} \otimes \tilde{Z}_{j}+\bar{\gamma}^{\prime k} \otimes \tilde{Z}_{j}^{\prime}, \\
\tau_{j k}= & \widetilde{R}_{\tilde{Z}_{j} \tilde{Z}_{k}^{\prime}}^{t}=\widetilde{R}_{\tilde{Z}_{k} \tilde{Z}_{j}^{\prime}}=-\bar{\gamma}^{j} \otimes \tilde{Z}_{k}^{\prime}+\bar{\gamma}^{\prime j} \otimes \tilde{Z}_{k}-\bar{\gamma}^{k} \otimes \tilde{Z}_{j}^{\prime}+\bar{\gamma}^{\prime k} \otimes \tilde{Z}_{j} .
\end{aligned}
$$

(If $n=2$, the operators $\sigma_{j k}$ and $\tau_{j k}$ do not appear, that is $\tilde{\mathfrak{h}}_{t}=\left\langle\rho_{0}, \rho_{1}, \varphi_{1}, \psi_{1}\right\rangle$, and if $n=1, \tilde{\mathfrak{h}}_{t}$ is generated by $\rho_{0}=\widetilde{R}_{\tilde{A} \tilde{U}}^{t}=2(t-3)(\bar{\alpha} \otimes \tilde{U}-\bar{\beta} \otimes \tilde{A})$.) The Lie structure of $\tilde{\mathfrak{g}}_{t}=\tilde{\mathfrak{h}}_{t}+\overline{\mathfrak{m}}$ is defined by the Eqs. (2.3). If $t \neq(2 n+1) / n$, the subalgebra $\tilde{\mathfrak{h}}_{t}$ is isomorphic to the Lie algebra $\mathfrak{k}=\mathfrak{s}(\mathfrak{u}(n)+\mathfrak{u}(1)) \cong \mathfrak{u}(n)$ in $\S 3.1$, via the map $h: \tilde{\mathfrak{h}}_{t} \rightarrow \mathfrak{k}$ given by $h\left(\rho_{0}\right)=2 U_{\mathfrak{k}}, h\left(\rho_{r}\right)=-\left(C_{r}+U_{\mathfrak{k}}\right), h\left(\varphi_{r}\right)=\left(Z_{r}\right)_{\mathfrak{k}}, h\left(\psi_{r}\right)=\left(Z_{r}^{\prime}\right)_{\mathfrak{k}}, h\left(\sigma_{j k}\right)=F_{j k}$, $h\left(\tau_{j k}\right)=-H_{j k}$. If we put $\hat{\rho}_{0}=\frac{1}{2}\left(\rho_{0}-2 \bar{\xi}\right), \hat{\rho}_{r}=-\frac{1}{2} \rho_{0}-\rho_{r}-\bar{\xi}$, then $\widehat{\mathfrak{s u}}(n, 1)=$ $\left\langle\hat{\rho}_{0}, \hat{\rho}_{r}, \varphi_{r}, \psi_{r}, \sigma_{j k}, \tau_{j k}, \tilde{A}, \tilde{U}, \tilde{Z}_{r}, \tilde{Z}_{r}^{\prime}: r, j, k=1, \ldots, n-1, j<k\right\rangle$ is an ideal of $\tilde{\mathfrak{g}}_{t}$, and the map $h$ extends to a Lie algebra isomorphism $\tilde{h}: \widehat{\mathfrak{s u}}(n, 1) \rightarrow \mathfrak{s u}(n, 1)=\mathfrak{k}+\mathfrak{p}$, given by $\tilde{h}\left(\hat{\rho}_{0}\right)=U_{\mathfrak{k}}, \tilde{h}\left(\hat{\rho}_{r}\right)=C_{r}, \tilde{h}\left(\varphi_{r}\right)=\left(Z_{r}\right)_{\mathfrak{k}}, \tilde{h}\left(\psi_{r}\right)=\left(Z_{r}^{\prime}\right)_{\mathfrak{k}}, \tilde{h}\left(\sigma_{j k}\right)=F_{j k}, \tilde{h}\left(\tau_{j k}\right)=-H_{j k}$, 
$\tilde{h}(\tilde{A})=A_{0}, \tilde{h}(\tilde{U})=U_{\mathfrak{p}}, \tilde{h}\left(\tilde{Z}_{r}\right)=\left(Z_{r}\right)_{\mathfrak{p}}, \tilde{h}\left(\tilde{Z}_{r}^{\prime}\right)=\left(Z_{r}^{\prime}\right)_{\mathfrak{p}}$. Moreover, $\tilde{\mathfrak{g}}_{t}$ is the semidirect product of $\widehat{\mathfrak{s u}}(n, 1)$ and the line generated by $\bar{\xi}$ under the homomorphism $\delta_{t}:\langle\bar{\xi}\rangle \rightarrow$ $\operatorname{Der}(\widehat{\mathfrak{s u}}(n, 1))$, given by $\delta_{t}(\bar{\xi})(\tilde{A})=(t-1) \tilde{U}, \delta_{t}(\bar{\xi})(\tilde{U})=(1-t) \tilde{A}, \delta_{t}(\bar{\xi})\left(\tilde{Z}_{r}\right)=(1-t) \tilde{Z}_{r}^{\prime}$, $\delta_{t}(\bar{\xi})\left(\tilde{Z}_{r}^{\prime}\right)=(t-1) \tilde{Z}_{r}$, and $\delta_{t}(\bar{\xi})\left(\left\langle\hat{\rho}_{0}, \hat{\rho}_{r}, \varphi_{r}, \psi_{r}, \sigma_{j k}, \tau_{j k}\right\rangle\right)=0$. So, we have

Proposition 3.11. The reductive decomposition associated to the homogeneous Sasakian structure $S^{t}, t \neq(2 n+1) / n$, on the total space of the line bundle $\bar{M} \rightarrow \mathbb{C H}(n)$ is $\tilde{\mathfrak{g}}_{t}=\tilde{\mathfrak{h}}_{t}+\overline{\mathfrak{m}}$, where $\tilde{\mathfrak{h}}_{t} \cong \mathfrak{s}(\mathfrak{u}(n)+\mathfrak{u}(1)) \cong \mathfrak{u}(n) \subset \mathfrak{s u}(n, 1)$, and $\overline{\mathfrak{m}}=\mathfrak{p}+\langle\bar{\xi}\rangle=$ $\left\langle A_{0}, U_{\mathfrak{p}},\left(Z_{r}\right)_{\mathfrak{p}},\left(Z_{r}^{\prime}\right)_{\mathfrak{p}}, \bar{\xi}: 1 \leqslant r \leqslant n-1\right\rangle$. Moreover, $\tilde{\mathfrak{g}}_{t}$ is the semidirect product $\tilde{\mathfrak{g}}_{t}=\langle\bar{\xi}\rangle \ltimes_{\delta_{t}} \mathfrak{s u}(n, 1)$, where $\delta_{t}(\bar{\xi})\left(A_{0}\right)=(t-1) U_{\mathfrak{p}}, \delta_{t}(\bar{\xi})\left(U_{\mathfrak{p}}\right)=(1-t) A_{0}, \delta_{t}(\bar{\xi})\left(\left(Z_{r}\right)_{\mathfrak{p}}\right)=$ $(1-t)\left(Z_{r}^{\prime}\right)_{\mathfrak{p}}, \delta_{t}(\bar{\xi})\left(\left(Z_{r}^{\prime}\right)_{\mathfrak{p}}\right)=(t-1)\left(Z_{r}\right)_{\mathfrak{p}}$, and $\delta_{t}(\bar{\xi})\left(\tilde{\mathfrak{h}}_{t}\right)=0$.

If $n \geqslant 2$ and $t=(2 n+1) / n$, then it is easy to see that $\rho_{0}=\rho_{1}+\cdots+\rho_{n-1}$, and we put $\tilde{\rho}_{r}=\frac{1}{2}\left(\rho_{0}+\rho_{r}\right), 1 \leqslant r \leqslant n-1$. In this case, $\tilde{\mathfrak{g}}_{\frac{2 n+1}{n}}=\tilde{\mathfrak{h}}_{\frac{2 n+1}{n}}+\overline{\mathfrak{m}}$ coincides with the reductive decomposition $\mathfrak{s u}(n, 1)=\mathfrak{k}^{\prime}+\mathfrak{m}^{\prime}$, where $\mathfrak{k}^{\prime}=[\mathfrak{k}, \mathfrak{k}] \cong \mathfrak{s u}(n)$, and $\mathfrak{m}^{\prime}=\mathfrak{p}+\langle\mathfrak{c}\rangle$, being $\mathfrak{c}$ the center of $\mathfrak{k}$, which is generated by the element $E_{J}$ such that $\operatorname{ad}_{E_{J}}: \mathfrak{p} \rightarrow \mathfrak{p}$ defines the complex structure of $\mathbb{C H}(n)$. In fact, we have the isomorphism $f: \frac{\tilde{\mathfrak{g}}_{2 n+1}}{n} \rightarrow \mathfrak{s u}(n, 1)$ given by $f\left(\tilde{\rho}_{r}\right)=\frac{1}{2}\left(U_{\mathfrak{k}}-C_{r}\right), f\left(\varphi_{r}\right)=\left(Z_{r}\right)_{\mathfrak{k}}, f\left(\psi_{r}\right)=\left(Z_{r}^{\prime}\right)_{\mathfrak{k}}$, $f\left(\sigma_{j k}\right)^{n}=F_{j k}, f\left(\tau_{j k}\right)=-H_{j k}, f(\tilde{A})=A_{0}, f(\tilde{U})=U_{\mathfrak{p}}, f\left(\tilde{Z}_{r}\right)=\left(Z_{r}\right)_{\mathfrak{p}}, f\left(\tilde{Z}_{r}^{\prime}\right)=\left(Z_{r}^{\prime}\right)_{\mathfrak{p}}$, and $f(\bar{\xi})=-\frac{n+1}{n} E_{J}=-\frac{1}{2 n}\left(C_{1}+\cdots+C_{n-1}+(n+1) U_{\mathfrak{k}}\right)$, and, in particular, $f\left(\tilde{\mathfrak{h}}_{\frac{2 n+1}{n}}\right)=\mathfrak{k}^{\prime}$ and $f(\overline{\mathfrak{m}})=\mathfrak{m}^{\prime}$. If $n=1$ and $t=3$, then $\rho_{0}=0$. In this case, $\tilde{\mathfrak{h}}_{3}=0, \mathfrak{k}^{\prime}=[\mathfrak{k}, \mathfrak{k}]=0$, $\mathfrak{c}=\left\langle E_{J}\right\rangle, E_{J}=\frac{1}{2} U_{\mathfrak{k}}, \tilde{\mathfrak{g}}_{3}=\{0\}+\overline{\mathfrak{m}}$ is the reductive decomposition $\mathfrak{s u}(1,1)=\{0\}+\mathfrak{m}^{\prime}$, where $\overline{\mathfrak{m}}=\langle\tilde{A}, \tilde{U}, \bar{\xi}\rangle, \mathfrak{m}^{\prime}=\left\langle A_{0}, U_{\mathfrak{p}}, U_{\mathfrak{k}}\right\rangle$, and $f: \tilde{\mathfrak{g}}_{3} \rightarrow \mathfrak{s u}(1,1)$ such that $f(\tilde{A})=A_{0}$, $f(\tilde{U})=U_{\mathfrak{p}}, f(\bar{\xi})=-U_{\mathfrak{k}}$. Hence, we have obtained

Proposition 3.12. The reductive decomposition associated to the homogeneous Sasakian structure $S^{t}$, with $t=(2 n+1) / n$, on the total space of the line bundle $\bar{M} \rightarrow \mathbb{C H}(n)$ is $\mathfrak{s u}(n, 1)=\mathfrak{k}^{\prime}+\mathfrak{m}^{\prime}$, where $\mathfrak{k}^{\prime}=[\mathfrak{k}, \mathfrak{k}] \cong \mathfrak{s u}(n)$, and $\mathfrak{m}^{\prime}=\mathfrak{p}+\mathfrak{c}, \mathfrak{c}=\left\langle E_{J}\right\rangle$ being the center of $\mathfrak{k}$.

Remark 3.13. The reductive decomposition $\mathfrak{s u}(n, 1)=\mathfrak{k}^{\prime}+\mathfrak{m}^{\prime}$ associated to the homogeneous Sasakian structure $S^{t}$, with $t=\frac{2 n+1}{n}$, provides the description of $\bar{M}$ as the homogeneous space $\widetilde{S U}(n, 1) / K^{\prime}$, where $\widetilde{S U}(n, 1)$ is the universal covering of $S U(n, 1)$, and $K^{\prime} \cong S U(n)$ is the connected subgroup of $\widehat{S U}(n, 1)$ whose Lie algebra is $\mathfrak{k}^{\prime} \cong \mathfrak{s u}(n)$. (In particular, if $n=1, \bar{M}$ is the universal covering space of $S l(2, \mathbb{R})$.) These spaces appear in the classification by Jiménez and Kowalski [16] of complete simply connected $\varphi$-symmetric Sasakian manifolds, and they are also Sasakian space forms (they have constant $\varphi$-sectional curvature -7 ). Notice that for a Sasakian manifold, the condition of being a locally symmetric space is too strong, because in this case it is a space of constant curvature (Okumura [23]). For this reason, Takahashi [25] introduced $\varphi$-symmetric spaces in Sasakian geometry as generalizations of Sasakian space forms. They are also analogues of Hermitian symmetric spaces. A $\varphi$-symmetric space is a complete connected regular Sasakian manifold $\bar{M}$ that fibers over a Hermitian symmetric space $M$ so that the geodesic involutions of $M$ lift to involutive automorphisms of the Sasakian structure on $\bar{M}$. Moreover, each complete simply connected $\varphi$-symmetric space is a naturally reductive homogeneous space (Blair and Vanhecke [5]). 


\section{Acknowledgments}

Partially supported by the Ministry of Science and Innovation, Spain, under Project MTM2008-01386.

\section{References}

[1] E. Abbena and S. Garbiero, Almost-Hermitian homogeneous structures, Proc. Edinb. Math. Soc. (2) 31 (1988) 375-395.

[2] W. Ambrose and I. M. Singer, On homogeneous Riemannian manifolds, Duke Math. J. 25 (1958) 647-669.

[3] D. E. BlaIR, Contact manifolds in Riemannian geometry (Springer, Berlin, 1976).

[4] D. E. BLAIR, Riemannian geometry of contact and symplectic manifolds (Birkhäuser, Boston, 2002).

[5] D. E. Blair and L. Vanhecke, New characterizations of $\varphi$-symmetric spaces, Kōdai Math. J. 10 (1987) 102-107.

[6] W. M. Boothby and H. C. Wang, On contact manifolds, Ann. of Math. (2) 68 (1958) $721-734$.

[7] M. Castrillón López, P. M. Gadea and A. F. Swann, Homogeneous structures on real and complex hyperbolic spaces, Illinois J. Math., to appear.

[8] D. Chinea And C. González, An example of an almost cosymplectic homogeneous manifold, in Differential geometry, Peñiscola, 1985, Lecture Notes in Mathematics 1209 (Springer, Berlin, 1986), pp. 133-142.

[9] A. Díaz Miranda and A. Reventós, Homogeneous contact compact manifolds and homogeneous symplectic manifolds, Bull. Sci. Math. (2) 106 (1982), no. 4, $337-350$.

[10] J. Dorfmeister And K. NAKAJima, The fundamental conjecture for homogeneous Kähler manifolds, Acta Math. 161 (1988), no. 1-2, 23-70.

[11] A. Fino, Almost contact homogeneous structures, Boll. Un. Mat. Ital. (7) 9-A (1995) 299-311.

[12] P. M. Gadea, A. Montesinos Amilibia and J. Muñoz Masqué, Characterizing the complex hyperbolic space by Kähler homogeneous structures, Math. Proc. Cambridge Phil. Soc. 27 (2000) 87-94.

[13] P. M. Gadea and J. A. Oubiña, Reductive homogeneous pseudo-Riemannian manifolds, Monatsh. Math. 124 (1997) 17-34.

[14] C. González and D. Chinea, Quasi-Sasakian homogeneous structures on the generalized Heisenberg group H(p,1), Proc. Amer. Math. Soc. 105 (1989) 173-184. 
[15] S. Helgason, Differential geometry, Lie groups, and symmetric spaces (Academic Press, New York, 1978).

[16] J. Jiménez AND O. Kowalski, The classification of $\varphi$-symmetric Sasakian manifolds, Monatsh. Math. 115 (1993) 83-98.

[17] V. F. KiRIČENKo, On homogeneous Riemannian spaces with an invariant structure tensor, Soviet Math. Dokl. 21 (1980) 734-737.

[18] S. Kobayashi and K. Nomizu, Foundations of differential geometry. Vol. I, II (Wiley, New York, 1963, 1969).

[19] T. Koda and Y. Watanabe, Homogeneous almost contact Riemannian manifolds and infinitesimal models, Boll. Un. Mat. Ital. B (7) 11-B (1997), Suppl., fasc. 2, $11-24$.

[20] K. Nomizu, Invariant affine connections on homogeneous spaces, Amer. J. Math. 76 (1954) 33-65.

[21] H. Nishino, Alternative $N=(4,0)$ superstring and $\sigma$-models, Phys. Lett. B355 (1995) $117-126$.

[22] K. Ogiue, On fiberings of almost contact manifolds, Kōdai Math. Sem. Rep. 17 (1965) 53-62.

[23] M. Okumura, Some remarks on spaces with a certain contact structure, Tôhoku Math. J. (2) 14 (1962) 135-145.

[24] K. Sekigawa, Notes on homogeneous almost Hermitian manifolds, Hokkaido Math. J. 7 (1978) 206-213.

[25] T. Takahashi, Sasakian $\varphi$-symmetric spaces, Tôhoku Math. J. (2) 29 (1997) 91113.

[26] F. Tricerri And L. VAnHecke, Homogeneous structures on Riemannian Manifolds (Cambridge Univ. Press, Cambridge, 1983).

[27] B. DE Wit And P. VAn Nieuwenhuizen, Rigidly and locally supersymmetric two-dimensional nonlinear $\sigma$-models with torsion, Nucl. Phys. B312 (1989) 58-94. 